\title{
ENDOGENOUS SELECTION OR TREATMENT MODEL ESTIMATION
}

\author{
Arthur Lewbel \\ Boston College \\ Revised January 2006
}

\begin{abstract}
In a sample selection or treatment effects model, common unobservables may affect both the outcome and the probability of selection in unknown ways. This paper shows that the distribution function of potential outcomes, conditional on covariates, can be identified given an observed variable $V$ that affects the treatment or selection probability in certain ways and is conditionally independent of the error terms in a model of potential outcomes. Selection model estimators based on this identification are provided, which take the form of simple weighted averages, GMM, or two stage least squares. These estimators permit endogenous and mismeasured regressors. Empirical applications are provided to estimation of a firm investment model and schooling effects on wages model.
\end{abstract}

Portions of this paper were previously circulated under other titles including, "Two Stage Least Squares Estimation of Endogenous Sample Selection Models."

JEL Codes: C14, C25, C13. Keywords: Sample Selection, Treatment Effects, Censoring, Semiparametric, Endogeneity, Instrumental Variables, Switching Regressions, Heteroscedasticity, Latent Variable Models, Ordered Choice, Investment, Returns to Schooling.

* This research was supported in part by the NSF, grant SES-9905010. I'd like to thank Yuriy Tchamourliyski for research assistance, and Hidehiko Ichimura, Edward Vytlacil, Jim Powell, Jim Heckman, Fabio Schiantarelli, Stacey Chen, Jinyong Hahn, Alberto Abadie, Shakeeb Khan, and anonymous referees for data and helpful comments. Arthur Lewbel, Department of Economics, Boston College, 140 Commonwealth Ave., Chestnut Hill, MA, 02467, USA. (617)-552-3678, lewbel@bc.edu, , http://www2.bc.edu/ lewbel/ 


\section{Introduction}

Assume that for a sample of individuals $i=1, \ldots, n$ we observe an indicator $D$ that equals one if an individual is treated, selected, or completely observed, and zero otherwise. If $D=1$ we observe some scalar or vector $P$, otherwise let $P=0$. Define $P^{*}$ to equal the observed $P$ when $D=1$, otherwise $P^{*}$ equals the value of $P$ that would have been observed if $D$ had equaled one, that is, either a counterfactual or an unobserved response. Then $P=P^{*} D$. We also observe covariates, though selection on observables is not assumed. Treatment or selection $D$ may be unconditionally or conditionally correlated with $P^{*}$, so $P^{*}$ and $D$ may depend on common unobservables. Rubin (1974) type restrictions like unconfoundedness or ignorability of selection are not assumed.

To illustrate, in a classic wage model (Gronau 1974, Heckman 1974, 1976), $D=1$ if the individual is employed, $P^{*}$ is the wage an individual would get if employed, and $P$ is the observed wage, which is zero for the unemployed. Both $P^{*}$ and $D$ depend on common unobservables such as ability, as well as on observable covariates such as measures of schooling or training.

Another example is models based on data sets where some regressors are missing, not at random. For example, models of individual's consumption or purchasing decisions depend on income $P^{*}$, and in surveys many individuals do not report their income. Failure to report income $(D=0)$ is likely to be correlated with income, even after conditioning on other observed covariates.

For simplicity, refer to $D$ as selection, though more generally it is just an indicator of not observing $P^{*}$ for whatever reason. Assume that selection $D$ is given by

$$
D=I\left(0 \leq M^{*}+V \leq A^{*}\right)
$$

where the unobserved $A^{*}$ can be a constant, a random variable, or infinity, $V$ is an observed, continuously distributed covariate (or known function of covariates) with large support, $M^{*}$ is an unobserved latent variable, and $I$ is the indicator function that equals one if its argument is true and zero otherwise. Typical parametric or semiparametric models of selection are special cases of equation (1) where $M^{*}$ is linear in covariates $X$ and a well behaved error term $e$, but that structure is not imposed here.

Setting the lower bound to zero in equation (1) is a free normalization, since no location assumptions are imposed on $M^{*}$ and $A^{*}$. Similarly, setting the coefficient of $V$ to one is (apart from sign) a free scale normalization that is imposed without loss of generality. 
In the wage model example, the typical assumption is that one chooses to work if the gains in utility from working, indexed by the latent $M^{*}+V$, are sufficiently large, so in that case $A^{*}$ is infinite. Examples in which $A^{*}$ is finite arise in ordered treatment or ordered selection models. For example, if an ordered choice model with latent variable $M^{*}+V$ determines an individual's years of schooling and $D$ indexes having exactly 12 years of schooling then individuals with $M^{*}+V<0$ choose 11 or fewer years while those with $M^{*}+V>A^{*}$ choose 13 or more years. We might then be interested in modelling the returns $P$ from having exactly 12 years of schooling. Examples of models like this with $A^{*}$ random include Cameron and Heckman (1998) and Carneiro, Hansen, and Heckman (2003).

A convenient feature of the proposed estimators is that they will not require specifying, modeling or estimating the $D$ (propensity score) model, apart from assuming equation (1) holds. For example, any dependence of $M^{*}$ on $X$ can be unknown and need not be estimated, and the estimator is the same regardless of whether $A^{*}$ is constant, random, or infinite. Empirical applications with both finite $A^{*}$ and infinite $A^{*}$ are provided.

Regarding outcomes, define $U^{*}$ and $U$ by

$$
\begin{aligned}
& \psi\left(P^{*}, X, V, \theta_{0}\right)=U^{*} \\
& \psi\left(P, X, V, \theta_{0}\right) D=U
\end{aligned}
$$

for some known vector valued function $\psi$. The initial goal will be estimation of $E\left(U^{*}\right)$ which in turn is used to estimate the parameter vector $\theta_{0}$. For example, if $\psi$ is defined by $P^{*}=U^{*}$, then an estimate of $E\left(U^{*}\right)=E\left(P^{*}\right)$ is an estimate of what the mean outcome in the population would be if everyone in the population were treated, selected, or observed. More generally, suppose that $\theta_{0}$ uniquely satisfies $E\left[\psi\left(P^{*}, X, V, \theta_{0}\right)\right]=0$. If there were no selection problem, so if $P^{*}$ instead of $P=P^{*} D$ was observable, then the generalized method of moments (GMM) could be applied, minimizing a quadratic form in the sample mean of $\psi\left(P^{*}, X, V, \theta_{0}\right)$, to consistently estimate $\theta_{0}$. In the presence of selection this GMM is infeasible, but it becomes feasible given an estimator of $E\left(U^{*}\right)$.

For example, let $X$ be the union of elements in vectors $Y$ and $Z$, where $Y$ is a vector of regressors and $Z$ is a vector of instruments. Suppose potential wages are determined by the model $P^{*}=Y^{\prime} \beta_{Y}+V \beta_{V}+\varepsilon$, where some of the regressors in $Y$ may be mismeasured, endogenous, or otherwise correlated with the error $\varepsilon$, and the error $\varepsilon$ could also have unspecified heteroskedasticity. Given ordinary instruments $Z$ that are uncorrelated with $\varepsilon$ and correlated with $V, Y$ ( $Z$ may include 
exogenous elements of $Y)$, define $\psi$ by $Z\left(P^{*}-Y^{\prime} \beta_{Y}-V \beta_{V}\right)=U^{*}$. If $P^{*}$ were observable for everyone then $\beta=\beta_{V}, \beta_{Y}$ could be estimated by GMM, an example of which is a linear two stage least squares regression of $P^{*}$ on $V, Y$ using instruments $Z$. The difficulty is that this estimator is infeasible because of the selection problem, that is, we only observe $P$ instead of $P^{*}$, and unobservables that determine the selection such as $M^{*}$ are correlated with $P^{*}$ and $U^{*}$. Feasible estimation of $\beta$ requires an estimator for $E\left(U^{*}\right)$.

Define the weighting scalar $W$ by

$$
W=\frac{D}{f(V \mid X)}
$$

where $f$ is the conditional probability density function of $V$ given $X$. This paper shows that under general conditions

$$
E\left(U^{*}\right)=\operatorname{plim}_{n \rightarrow \infty} \frac{\sum_{i=1}^{n} U_{i} W_{i}}{\sum_{i=1}^{n} W_{i}}
$$

so $E\left(U^{*}\right)$ is consistently estimated as the weighted average of the observed $U_{i}$ (including $U_{i}=0$ for all unobserved outcomes) using weights $W$. Based on equation (3), the infeasible GMM for estimating $\theta_{0}$ is converted into a feasible GMM using the observable $W \psi\left(P, X, V, \theta_{0}\right)$ in place of the unobservable function $\psi\left(P^{*}, X, V, \theta_{0}\right)$. In the above two stage least squares example, this means $\beta$ would be consistently estimated by an ordinary linear two stage least squares regression of $W P$ on $W V, W Y$ using instruments $Z$.

The main assumptions required for equation (3) to hold are that the support of $V \mid X$ contains the supports of both $-M^{*} \mid X$ and $A^{*}-M^{*} \mid X$ (these could all equal the real line, for example), and that

$$
V\left|X, U^{*}, M^{*}, A^{*} \sim V\right| X
$$

that is, $V$ is conditionally independent of the unobserved latent variables of the model, conditioning on the set of covariates $X$.

To give some intuition for equation (3), and why the above restrictions on $V$ are required, suppose for the moment that $A^{*}=a$ is constant and that $V$ has a uniform distribution with constant density $f$, independent of $M^{*}, U^{*}$. In that case we would have $E(U)=E\left[E\left(I\left(0 \leq M^{*}+V \leq a\right) U^{*} \mid M^{*}, U^{*}\right)\right]=$ $E\left(\int_{-M^{*}}^{a-M^{*}} U^{*} f d v\right)=a E\left(U^{*}\right)$ and similarly $E(D)=a$, so in that case there would be no selection problem (or more precisely, unconditional propensity score 
weighting would then fix the selection problem), because we would then have $E\left(U^{*}\right)=E(D U) / E(D)$. The key is that $M^{*}$, the source of unobserved correlation between $D$ and $U^{*}$, drops out when $V$ is independently, uniformly distributed. In the general problem, $V$ is not an independent uniform, but weighting by $W$, and hence scaling by the conditional density of $f$, is algebraically equivalent to converting $V$ to a uniform. Given equation (4), it suffices to condition the density of $V$ on the observable $X$, and the support assumption on $V$ ensures the bounds of the integral are not cut off.

Equation (3) resembles propensity score weighting estimators (see, e.g., Horvitz and Thompson (1952), Koul, Susarla, and van Ryzin (1981), Hahn (1998), and Hirano, Imbens and Ridder (2003)), but equation (3) holds even though the independence and conditioning assumptions required for consistency of propensity score weighted estimators are not imposed here. Specifically, we cannot use $E(U) / E(D)$ or $E[E(U \mid V, X) / E(D \mid V, X)]$ to estimate $E\left(U^{*}\right)$, because $D$ and $U^{*}$ (or equivalently, $M^{*}$ and $U^{*}$ ) can covary, even after conditioning on observables like $X, V$. The unconfoundedness assumption is not satisfied. However, averaging after $V$ density weighting is equivalent to integrating over $V$, that is equation (3) implies $E\left(U^{*}\right)=E\left[\int_{-\infty}^{\infty} E(U \mid V, X) d V\right] / E\left[\int_{-\infty}^{\infty} E(D \mid V, X) d V\right]$ when these expectations exist, so the proposed estimator is equivalent to weighting an integral of the conditional expectation of $U$ by an integral of the conditional propensity score.

One interpretation of equation (4) is simply that $V$ is an exogenous covariate, in the strong sense of being conditionally (conditioning on other covariates $X$ ) independent of the unobservables in the model, and hence conditionally independent of the errors if the model were parameterized. More generally, equation (4) is an example of an exclusion restriction, of the sort that is commonly used to identify models in simultaneous systems. Section 2.5 of Powell's (1994) survey discusses the use of exclusion assumptions in semiparametric estimators. Magnac and Maurin (2003) call this a partial independence assumption. In models where the errors are independent of regressors, every regressor is exogenous and so satisfies equation (4). Blundell and Powell (2004) and Heckman and Vytlacil (2004) use exclusion assumptions for identification in binary choice and treatment models.

Requiring a regressor to have support containing a large or infinite interval, encompassing the supports of other variables, is also common in the semiparametric limited dependent variable model literature. Examples include Manski (1975,1985), Han (1987), Horowitz (1992), and Cavanagh and Sherman (1998).

The estimator here weights observations by the density of a regressor $V$ that 
satisfies exclusion and large support assumptions. Lewbel $(1998,2000)$ and Khan and Lewbel (2005) use a similar idea to estimate linear index, binary choice and truncated regression models, respectively. Magnac and Maurin (2003) prove semiparametric efficiency of Lewbel (2000), and Jacho-Chavez (2005) shows semiparametric efficiency of a general class of density weighted estimators. Magnac and Maurin (2003) also show in the binary choice context that large support can be replaced by a tail symmetry restriction, and that identification based on either is observationally equivalent. Lewbel, Linton, and McFadden (2002) extend and apply the binary choice estimator in Lewbel (2000) to recover general features of a distribution from binary outcomes, with application to contingent valuation and willingness to pay studies. Other empirical applications of the methodology in discrete choice applications include Anton, Fernández, and Rodriguez-Póo (2001) and Cogneau and Maurin (2001). The latter analyze enrollment of children into school in Madagascar, using the date of birth of the child within the relevant year as the regressor $V$ that satisfies exclusion and large support.

No restriction is placed on the joint distribution of $M^{*}$ and $U^{*}$ other than equation (4). In the wage example, this means that no restriction is placed on the joint distribution of unobservables such as ability that determine employment status and wages, other than that these unobservables are (conditionally on $X$ ) independent of the one covariate $V$. This is a markedly weaker restriction on the joint distribution of outcomes and selection than is required by other selection or treatment estimators. In particular, the estimators proposed here do not assume unconfoundedness, selection on observables, independence of errors and covariates, or any parameterization of the joint distribution of selection and outcome errors. The assumptions permit general forms of endogeneity, mismeasurement, and heteroskedasticity in both selection and outcomes.

Estimation based on equation (3) is consistent whether $A^{*}$ in equation (1) is finite or infinite. The estimator is numerically the same in either case. When $A^{*}$ is finite the estimator has an ordinary root $n$ limiting distribution. However, when $A^{*}$ and the support of $M^{*}$ are both infinite, then the identification is only at infinity, as in Heckman (1990) and Andrews and Schafgans (1998). If $A^{*}$ is infinite and the support of $V \mid X$ is bounded, then the estimator can still be used and will again have an ordinary root $n$ limiting distribution, though in this case the estimator will be asymptotically biased, with bias of order $1 / \tau$ where $\tau$ is the largest value $V$ can take on. Since $\tau$ can be arbitrarily large this bias, when it is present, can be arbitrarily small. In particular, with a finite sample size one could never tell if $V$ really had infinite support, or if it had bounded support with $\tau$ large enough to make the resulting bias smaller than any computer roundoff error, or any number 
of significant digits one chose for reporting estimates.

Many estimators exist for treatment, sample selection, missing data, and other related models. Standard maximum likelihood estimation requires that the entire joint distribution of the unobservables, conditional on covariates or instruments, be finitely parameterized. In particular, the selection equation (and the endogenous regressors as functions of instruments) would need to be completely specified. Alternative assumptions like unconfoundedness or selection on observables likewise impose strong restrictions on the joint behavior of unobservables affecting selection and outcomes.

Related parametric estimators of sample selection models include Horvitz and Thompson (1952), Heckman (1974, 1976, 1979, 1990), Rubin (1974), Koul, Susarla, and van Ryzin (1981), Lee (1982), and Rosenbaum and Rubin (1985).

Related semiparametric estimators of sample selection, and other probability weighted models, include Newey (1988), (1999), Cosslett (1991), Ichimura and Lee (1991), Lee (1992, 1994), and Ahn and Powell (1993), Angrist and Imbens (1995), Donald (1995), Wooldridge (1995), Kyriazidou (1997), Andrews and Schafgans (1998), Hahn (1998), Chen and Lee (1998), Abadie (2001), Hirano, Imbens and Ridder (2003), and Das, Newey, and Vella (2003). Surveys include Wainer (1986), Manski (1994), and Vella (1998).

\section{Mean Estimation}

Selection or treatment is determined by the model $D=I\left(0 \leq M^{*}+V \leq A^{*}\right)$. The following lemma gives an alternate characterization for the case where $A^{*}$ is infinite. Proofs of Lemmas, Theorems, and Corollaries are in the Appendix.

LEMMA 1. If $\operatorname{pr}(D=1 \mid V, X)$ is strictly monotonically increasing in $V$, then there exists an $M^{*}$ such that $E(D \mid V, X)=E\left[I\left(0 \leq M^{*}+V\right) \mid V, X\right]$ and $V \perp X, M^{*}$.

Lemma 1 shows that the assumption that selection is determined by a model of the form $D=I\left(0 \leq M^{*}+V\right)$ with an independent $M^{*}$ is observationally equivalent to just assuming that, conditional on $V, X$, the probability of selection is monotonically increasing in $V$. Closely related equivalence results are Vytlacil (2002) and Magnac and Maurin (2003). In this case where $A^{*}$ is infinite, $D$ equals one with probability approaching one as $V$ goes to infinity, which provides identification at infinity for the outcome model. 
When $A^{*}$ is finite, the probability of selection is not monotonic in $V$. Instead, this probability goes to zero for both small and large $V$, so in this case the outcome model is not identified at infinity.

Assumption A1. $D$ is a binary treatment or selection indicator, $X$ is a covariate vector, and $V$ is a covariate scalar. $U^{*}$ is an unobserved random vector with finite mean. $U=U^{*} D$. The indicator $D$ is determined by $D=$ $I\left(0 \leq M^{*}+V \leq A^{*}\right)$, where $A^{*}$ and $M^{*}$ are unobserved latent variables and $E\left(U^{*} \mid A^{*}\right)=E\left(U^{*}\right)$. The random scalar $V$ is continuously distributed conditional on $X . V\left|X, U^{*}, M^{*}, A^{*} \sim V\right| X .0<E(D)<1$. Either $E\left(A^{*}\right)$ is finite or $E\left(M^{*} U^{*}\right)$ and $E\left(M^{*}\right)$ are finite.

Let $f(V \mid X)$ denote the probability density function of $V$ given $X$ and define $W=D / f(V \mid X)$.

Assumption A2. The support of $V \mid X$ is an interval on the real line and contains the supports of $-M^{*} \mid X$ and $A^{*}-M^{*} \mid X$.

Define the estimator

$$
\widehat{\mu}=\frac{\sum_{i=1}^{n} U_{i} W_{i}}{\sum_{i=1}^{n} W_{i}}
$$

TheOrem 1. Let Assumptions A1 and A2 hold. Given $n$ independent, identically distributed draws of $U_{i}, W_{i}$,

$$
E\left(U^{*}\right)=\operatorname{plim} \widehat{\mu}
$$

The proof given for Theorem 1 in the appendix separately considers the three cases where $E\left(A^{*}\right)$ is finite (which of course includes the case where $A^{*}$ is a constant), where $A^{*}$ is infinite, and other cases such as $A^{*}$ infinite with probability between zero and one, as would arise if some individuals have $D=I\left(0 \leq M^{*}+\right.$ $\left.V \leq A^{*}\right)$ for finite $A^{*}$ while others have $D=I\left(0 \leq M^{*}+V\right)$. A more concise proof combining these cases is possible, but intermediate results in the proof as provided are used later.

Theorem 1 provides a consistent estimator of the mean of $P^{*}$ when $P^{*}$ is (conditional on $X$ ) independent of $V$, by letting $P^{*}=U^{*}$. This conditional independence can be rather restrictive, so later extensions relax this, instead imposing 
only that the errors in a model that includes $P^{*}$ be conditionally independent of $V$.

Theorem 1 immediately implies identification of the entire distribution function of $U^{*}$ (and hence of $P^{*}$ under conditional independence of $V$ ) because, for any constant $c$, Theorem 1 can be applied replacing $U_{i}^{*}$ with $I\left(U_{i}^{*} \leq c\right)$ and replacing $U_{i}$ with $I\left(U_{i} \leq c\right) D_{i}$, from which it then follows that

$$
\operatorname{plim} \frac{\sum_{i=1}^{n} I\left(U_{i} \leq c\right) W_{i}}{\sum_{i=1}^{n} W_{i}}=E\left[I\left(U_{i}^{*} \leq c\right)\right]=\operatorname{prob}\left(U_{i}^{*} \leq c\right) .
$$

Similarly, Theorem 1 can also be used to directly estimate any moments of $U^{*}$ by replacing $U_{i}^{*}$ and $U_{i}$ with $\left(U_{i}^{*}\right)^{c}$ and $\left(U_{i}\right)^{c}$ respectively.

Assumption A3. The support of $V \mid X$ contains the interval $(-\tau, \tau)$ for some positive constant $\tau$.

Corollary 1. Let Assumptions A1 and A3 hold. Then, given $n$ independent, identically distributed draws of $U_{i}, W_{i}$, plim $\widehat{\mu}=E\left(U^{*}\right)+O\left(\tau^{-1}\right)$.

Assumption A2 cannot be directly tested since $M^{*}$ is unobserved, but Corollary 1 shows that as long as the observable $V$ has a large support as defined by Assumption A3 for large $\tau$, the estimator will have at most a small asymptotic bias even if the support of $V$ is not large enough to satisfy Assumption A2.

Assumption A4. sup $(\operatorname{supp}(W))$ and $E\left(U^{* 2}\right)$ are finite. Define $\mu=$ plim $\widehat{\mu}$.

Assumption A4 implies that the means and variances of $W$ and $U W$ are finite.

Corollary 2. Let Assumptions A1 and A4 hold. Then, given $n$ independent, identically distributed draws of $U_{i}, W_{i}$,

$$
\sqrt{n}(\widehat{\mu}-\mu) \stackrel{d}{\longrightarrow} N\left(0, \frac{\operatorname{var}[(U-\mu) W]}{E(W)^{2}}\right) .
$$

Corollary 2 shows that with finite fixed or random $A^{*}$, the estimator $\widehat{\mu}$ is $\operatorname{root} n$ consistent and asymptotically normal. Assumption A4 conflicts with Assumption A2 when $A^{*}$ is infinite. However, by Corollary 1 , if we limit $V$ to a very large but not infinite support, the resulting asymptotic bias $\mu-E\left(U^{*}\right)$ will be tiny. If $V$ has 
large enough bounded support, this bias can be made smaller than any computer roundoff error, while still preserving Assumption A4 and hence a root $n$ normal limiting distribution.

The difficulty with allowing $A^{*}$ to be infinite while satisfying Assumption A2 (which would then require the upper bound on the support of $V$ to be infinite) is not only that the rate of convergence of $\widehat{\mu}$ becomes slower than root $n$, but also that the Lindeberg condition for asymptotic normality at any rate will generally be violated. This problem could be overcome using asymptotic trimming, replacing the weights $W_{i}$ in the estimator with $W_{\tau i}$ as defined in the proof of Theorem 1, and letting $\tau \rightarrow \infty$ at an appropriate rate (slower than the rate in the proof of Theorem 1, and hence using an estimator that is not asymptotically equivalent to $\widehat{\mu})$. The resulting estimator would then be essentially equivalent to the Andrews and Schafgans (1998) identification at infinity estimator (using $f$ in place of their weighting function), and so is not pursued further here. So, although the estimator can be consistent even when $A^{*}$ is infinite by Theorem 1 , for the sake of obtaining simple limiting distributions, avoiding asymptotic trimming, exploiting Corollary 1 in place of the untestable Assumption A2, and avoiding duplication of existing identification at infinity estimators, Assumption A4 will be maintained for the remainder of this paper, with the understanding that the estimand will therefore suffer from an arbitrarily small asymptotic bias in applications where $A^{*}$ is infinite.

\section{GMM Estimation}

Assumption A5. Let $P$ be an observed outcome satisfying $P=P^{*} D$ for some latent, unobserved $P^{*}$. Let $U^{*}=\psi\left(P^{*}, X, V, \theta^{*}\right)$ and $U=\psi\left(P, X, V, \theta^{*}\right) D$, where the vector valued function $\psi(P, X, V, \theta)$ is known and continuously differentiable in a parameter vector $\theta$. Define $\Theta$ to be the set of possible values of $\theta$ and $\Omega$ to be a positive definite matrix. Among all $\theta \in \Theta, E\left[\psi\left(P^{*}, X, V, \theta\right)\right]=0$ only if $\theta=\theta^{*}$. For all $\theta \in \Theta, v\left(\theta_{0}\right)^{\prime} \Omega v(\theta)$ is nonsingular, where $v(\theta)$ and $\theta_{0}$ are given by

$$
\begin{gathered}
\nu(\theta)=E\left(W \frac{\partial \psi(P, X, V, \theta)}{\partial \theta}\right) / E(W) \\
\theta_{0}=\arg \min _{\theta \in \Theta} E[W \psi(P, X, V, \theta)]^{\prime} \Omega E[W \psi(P, X, V, \theta)]
\end{gathered}
$$


Theorem 2. Let Assumptions A1, A3, A4, and A5 hold. If $\mu=E\left(U^{*}\right)$ then $\theta_{0}=\theta^{*}$, and if $\mu=E\left(U^{*}\right)+O\left(\tau^{-1}\right)$ then $\theta_{0}=\theta^{*}+O\left(\tau^{-1}\right)$.

Corollary 3. Let Assumptions A1, A3, A4, and A5 hold. Assume $n$ independent, identically distributed draws of $W_{i}, P_{i}, X_{i}, V_{i}$. Assume $\Theta$ is compact and $\theta_{0}$, which is uniquely defined by equation (8), is in the interior of $\Theta$. Assume the second moment of $W \psi(P, X, V, \theta)$ is finite and that $W \partial \psi(P, X, V, \theta) / \partial \theta$ is bounded in absolute value by a function $b(W, P, X, V)$ that has finite mean. Let $\Omega_{n}$ be a sequence of positive definite matrices with $p \lim \Omega_{n}=\Omega$. Define $\widehat{\theta}$ by

$$
\widehat{\theta}=\arg \min _{\theta \in \Theta}\left[\sum_{i=1}^{n} W_{i} \psi\left(P_{i}, X_{i}, V_{i}, \theta\right)\right]^{\prime} \Omega_{n}\left[\sum_{i=1}^{n} W_{i} \psi\left(P_{i}, X_{i}, V_{i}, \theta\right)\right]
$$

Then

$$
\sqrt{n}\left(\widehat{\theta}-\theta_{0}\right) \stackrel{d}{\longrightarrow} N\left(0,\left(S_{0}^{\prime} \Omega S_{0}\right)^{-1} S_{0}^{\prime} \Omega \Sigma_{0} \Omega S_{0}\left(S_{0}^{\prime} \Omega S_{0}\right)^{-1}\right) .
$$

where $S_{0}=E\left[W \partial \psi\left(P, X, V, \theta_{0}\right) / \partial \theta\right]$ and $\Sigma_{0}=E\left[W^{2} \psi\left(P, X, V, \theta_{0}\right) \psi\left(P, X, V, \theta_{0}\right)^{\prime}\right]$.

Efficiency is obtained in the usual way by two step GMM, constructing $\Omega_{n}$ so that $\Omega=\Sigma_{0}^{-1}$.

As discussed in the introduction, if there were no selection problem so $P^{*}$ could be observed, $\theta$ would be estimated by applying GMM to the moments $E\left[\psi\left(P^{*}, X, V, \theta^{*}\right)\right]=0$. This estimator is infeasible, but Theorem 2 and Corollary 3 describe the corresponding feasible GMM that replaces $P^{*}$ with the observable $P$ and corrects for selection by multiplying the moments by $W$, i.e., using the feasible moments $E\left[W \psi\left(P, X, V, \theta_{0}\right)\right]=0$. The resulting estimand $\theta_{0}$ equals the desired $\theta^{*}$ when $A^{*}$ has finite mean and the support of $V$ is sufficiently large, otherwise the difference between $\theta_{0}$ and $\theta^{*}$ (the asymptotic bias) is of order $O\left(\tau^{-1}\right)$ where $\tau$ is the largest value $V$ can take on. As discussed earlier, this bias can be assumed to be smaller than any printed coefficient roundoff error, by a support assumption that could never be falsified with a finite data set. Of course the real question, addressed later in the empirical applications and Monte Carlo, will be whether the above root $n$ limiting distribution theory provides a good approximation to the distribution of $\widehat{\theta}$.

\section{Example Models}

The example models in this section will all be consistent with the assumptions of Theorem 2. These models use the following definitions and assumptions. Let $Z$ be 
a vector of covariates, which are exogenous in the sense that they are uncorrelated with the errors in a model of $P^{*}$. Assume $Z$ does not include $V$ (more generally, $V$ is not a deterministic function of $Z$ ). Let $Y$ be a vector of covariates, some of which may be endogenous, in that they may be correlated with the errors in a model of $P^{*}$. The vector $Y$ can include elements of $Z$. Define $X$ to be the union of all the elements of $Z$ and $Y$. The data consist of a sample of observations of $X, V, P, D$, which implies that $Z$ and $Y$ are also observed.

\subsection{Examples of Selection Models}

This paper's estimators assume equation (1) holds, but they do not require specifying, modeling or estimating the resulting probability of selection (propensity score). However, as an illustration consider $D=I\left(a_{0}+e_{0} \leq M(X, e)+V \leq\right.$ $\left.a_{1}+e_{1}\right)$ with errors $e_{1}, e_{0}$, and $e$ and unknown function $M$. Then equation (1) holds with $A^{*}=a_{1}-a_{0}+e_{1}-e_{0}$ and $M^{*}=-a_{0}+M(X, e)+e_{0}$. This is a random thresholds ordered selection model. For example, $M(X, e)$ could equal the benefits of college and $-V$ could be a measure of the cost of college. If benefits minus cost for an individual are low, below $a_{0}+e_{0}$, then the individual does not get a college degree, while if the benefits are very high, above $a_{1}+e_{1}$, then the individual goes on to graduate school. $D$ would then the indicator of just getting a college degree, and the associated $P^{*}$ could be some outcome like earnings associated with getting just a college degree. The possible randomness in the thresholds, $e_{0}$ and $e_{1}$, could embody unobserved heterogeneity in the utility of education.

More common models like fixed threshold ordered choice $D=I\left(a_{0} \leq X^{\prime} \beta+\right.$ $\left.V+e \leq a_{1}\right)$ or standard binary choice $D=I\left(0 \leq X^{\prime} \beta+V+e\right)$, are special cases that are included in this general framework. The estimator allows covariates other than $V$ to be endogenous, and errors to be heteroskedastic. For example, in all of the above models $X$ could be endogenous or mismeasured, with the joint distribution of $e_{1}, e_{0}, e, X$ unknown. More generally, the estimator does not require modeling or estimating the dependence of $M^{*}$ on $X$, and the estimator is the same regardless of whether $A^{*}$ is constant, random, or infinite. Empirical applications with both finite $A^{*}$ and infinite $A^{*}$ are provided.

\subsection{Examples of Outcome Models}

Suppose for a known function $h$ that

$$
h\left(P^{*}, Y, V, \beta\right)=\varepsilon, \quad E(\varepsilon Z)=0, \quad \varepsilon|V, X \sim \varepsilon| X
$$


Some or all of the elements of $P^{*}, Y$ may be endogenous and hence correlated with $\varepsilon$, so estimation is based on $E(\varepsilon Z)=0$, that is, if $P^{*}$ were observed the parameters $\beta$ would be estimated by applying GMM to the moments $E\left[Z h\left(P^{*}, Y, V, \beta\right)\right]=$ 0 . The unobservables that affect selection $D$ (that is, $M^{*}$ and $A^{*}$ ) can be correlated in unknown ways with $\varepsilon$. This model fits the assumptions of Theorem 2 by defining the function $\psi$ as

$$
\psi\left(P^{*}, X, V, \theta\right)=Z h\left(P^{*}, Y, V, \beta\right) .
$$

For example, $h\left(P^{*}, Y, V, \beta\right)=P^{*}-H(Y, V, \beta)$ could be a model of wages $P^{*}$ where $Y$ includes some endogenous regressors, e.g., spouse's income or transfers from parents, and $V$ (which helps determine labor force participation $D$ ) can be regressor in the $h$ model.

Another example is $h\left(P^{*}, Y, V, \beta\right)=Y-H\left(P^{*}, Z, V, \beta\right)$, which could be a model of consumption of a vector of goods $Y$ where $P^{*}$ is income that is not reported by a significant number of individuals in the sample, and where income nonresponse is correlated with $\varepsilon$, even after conditioning on observables.

Another class of models that can estimated using Corollary 3 are models that could have been estimated by maximum likelihood if $P^{*}$ were observed. For example, suppose that

$$
P^{*}=H(X, \beta, \varepsilon), \quad \varepsilon|V, X \sim \varepsilon| X, \varepsilon \sim F_{\varepsilon}(\varepsilon \mid X, \delta)
$$

So $H$ is a known parametric model for $P^{*}$ having latent errors $\varepsilon$, The errors $\varepsilon$ are conditionally independent of $V$ given $X$ (so $V$ is exogenous), and the conditional distribution function of $\varepsilon$ given $X$, denoted by $F_{\varepsilon}$, is known up to a parameter vector $\delta$. The unobservables that affect $D$ can be correlated in unknown ways with $\varepsilon$. Let $\theta=(\beta, \delta)$. Assuming each $\varepsilon \mid X$ is independently and identically distributed, we can construct a corresponding log likelihood function $\sum_{i=1}^{n} L\left(P_{i}^{*}, X_{i}, \theta\right)$ that could be used to estimate $\theta$ by maximum likelihood if $P^{*}$ were observable. Given ordinary maximum likelihood regularity, define the function $\psi$ by $\psi\left(P^{*}, X, V, \theta\right)=\partial L\left(P^{*}, X, \theta\right) / \partial \theta$, (the score function) and $\theta$ would be identified from $E\left[\psi\left(P^{*}, X, V, \theta_{0}\right)\right]=0$. Corollary 3 can then be used to estimate $\theta$ given $P$ instead of $P^{*}$

For an example of model (10), suppose that

$$
P^{*}=I\left(\beta^{\prime} X+\varepsilon \geq 0\right), \quad \varepsilon \perp V, X, \varepsilon \sim N(0,1)
$$

that is, the unobserved outcome $P^{*}$ is determined by a probit model. Equivalently, we may interpret this model as one where an individual makes two binary decisions or choices, $D$ and $P^{*}$, and we can only observe the outcome of the second 
decision, $P^{*}$, when the first decision is $D=1$. The unobservables that affect both decisions are related in unknown ways, so we do not know the joint distribution of $\varepsilon$ and errors in the $D$ model, nor do we know how those errors jointly vary with $X$. It is only the marginal distribution of $\varepsilon$ that is specified here. In this example $\psi\left(P^{*}, X, V, \beta\right)$ would just be the ordinary probit score function for each observation of this $P^{*}$. As this example shows, we do not require continuity of $P^{*}$, so the methodology can be used without change when the unobserved potential outcome $P^{*}$ is discrete, censored, or otherwise limited.

The main impact of the exclusion restriction (4) for the $P^{*}$ model is the implication that $U^{*}\left|V, X \sim U^{*}\right| X$ for $U^{*}=\psi\left(P^{*}, X, V, \theta\right)$. In the above examples, the assumption that either $\varepsilon|V, X \sim \varepsilon| X$ or $\varepsilon \perp V, X$ ensures that this requirement is satisfied.

\subsection{Examples of Density Models}

The GMM estimator in Corollary 3 assumes $W$ and therefore the density $f(V \mid$ $X)$ is known. Estimation remains straightforward if $f(V \mid X)$ is finitely parameterized. In this case, denote the conditional density of $V$ as $f(V \mid X, \lambda)$, and let the vector $\theta$ include both the set of unknown parameters in the $P^{*}$ model $\psi$ and the parameters $\lambda$ of the distribution of $f$. Let $R(V, X, \theta)$ be any vector valued function having the property that $\lambda$ is identified from the moments $E[R(V, X, \theta)]=0$. In particular, we could let

$$
R(V, X, \theta)=\frac{\partial \ln f(V \mid X, \lambda)}{\partial \lambda}
$$

in which case $R(V, X, \theta)$ is the score function associated with the maximum likelihood estimator of the parameters of $f$. Estimation of the model then proceeds by applying GMM to the set of moments

$$
E\left(\begin{array}{c}
\psi(P, X, V, \theta) D / f(V \mid X, \theta) \\
R(V, X, \theta)
\end{array}\right)=0
$$

and standard GMM limiting distribution theory applies. See, e.g., Newey (1984) or Wooldridge (2001), p. 425.

For example, suppose that we can model $V$ in terms of other covariates as

$$
V=g(X, \alpha)+\sigma(X, \gamma) \eta, \quad \eta \perp X, \varepsilon
$$

for some known functions $g$ and $\sigma$ with $\sigma(X, \gamma)>0$, unknown parameter vectors $\alpha$ and $\gamma$, and unobserved error term $\eta$ having some known density function $f_{\eta}$ with 
mean zero and variance one. We may think of $\eta$ as an exogenous covariate in the $P^{*}$ model. Then

$$
f(V \mid X)=\frac{1}{\sigma(X, \gamma)} f_{\eta}\left(\frac{V-g(X, \alpha)}{\sigma(X, \gamma)}\right)
$$

and equation (11) then becomes

$$
E\left(\begin{array}{c}
\frac{\sigma(X, \gamma) \psi(P, X, V, \theta) D}{f_{\eta}([V-g(X, \alpha)] / \sigma(X, \gamma))} \\
V-g(X, \alpha) \\
{[V-g(X, \alpha)]^{2}-\sigma(X, \gamma)^{2}}
\end{array}\right)=0
$$

where $\theta$ (which includes $\alpha$ and $\gamma$ along with whatever parameters appear in the model $\psi$ ) is estimated by applying standard GMM to this set of moments. A leading case would be taking $f_{\eta}$ to be standard normal, though other (in particular more flexible classes of densities), could also be used.

If $f(V \mid X)$ is not finitely parameterized, the GMM estimator can still be applied by replacing $W=D / f(V \mid X)$ with $\widehat{W}=D / \widehat{f}(V \mid X)$ in Corollary 3, where $\widehat{f}(V \mid X)$ is a nonparametric estimator of $f(V \mid X)$, such as a kernel density estimator. The general limiting distribution theory for these types of semiparametric two step estimators (where the first step is a nonparametric plug in like this) is given by Newey and McFadden (1984). The relevant result is that, with sufficient regularity,

$$
\begin{aligned}
& \sqrt{n}\left[\frac{1}{n} \sum_{i=1}^{n} \frac{T_{i}}{\widehat{f}\left(V_{i} \mid X_{i}\right)}-E\left(\frac{T}{f(V \mid X)}\right)\right] \\
& \stackrel{d}{\longrightarrow} N\left(0, \operatorname{var}\left[\frac{T}{f(V \mid X)}+E\left(\frac{T}{f(V \mid X)} \mid X\right)-E\left(\frac{T}{f(V \mid X)} \mid V, X\right)\right]\right)
\end{aligned}
$$

where $T$ is a random vector. Khan and Lewbel (2005) provide one example of detailed regularity conditions that suffice, including asymptotic trimming to deal with boundary effects in the kernel estimation of $f$ in the denominator. They assume the density is bounded away from zero except in the neighborhood of the boundary of the data, and trim by excluding observations that are within a given distance from this boundary, letting that distance decrease at an appropriate rate as the sample size grows. They also show that with retangular support one can more simply trim by dropping the observations where each continuous covariate takes on its smallest and biggest values in the sample. 
To apply this result to the GMM estimator, let $T=D \psi(P, X, V, \theta)$ to obtain the limiting distribution of the sample average of $\widehat{W} \psi(P, X, V, \theta)$. The result is if $\widehat{\theta}$ is given by

$$
\widehat{\theta}=\arg \min _{\theta \in \Theta}\left[\sum_{i=1}^{n} \frac{D_{i} \psi\left(P_{i}, X_{i}, V_{i}, \theta\right)}{\widehat{f}\left(V_{i} \mid X_{i}\right)}\right]^{\prime} \Omega_{n}\left[\sum_{i=1}^{n} \frac{D_{i} \psi\left(P_{i}, X_{i}, V_{i}, \theta\right)}{\widehat{f}\left(V_{i} \mid X_{i}\right)}\right]
$$

with independent, identically distributed observations, then

$$
\sqrt{n}\left(\widehat{\theta}-\theta_{0}\right) \stackrel{d}{\longrightarrow} N\left(0,\left(S_{0}^{\prime} \Omega S_{0}\right)^{-1} S_{0}^{\prime} \Omega \widetilde{\Sigma}_{0} \Omega S_{0}\left(S_{0}^{\prime} \Omega S_{0}\right)^{-1}\right)
$$

where $\Omega$ and $S_{0}$ are as before and

$$
\widetilde{\Sigma}_{0}=\operatorname{var}\left[\frac{D \psi(P, X, V, \theta)}{f(V \mid X)}+E\left(\frac{D \psi(P, X, V, \theta)}{f(V \mid X)} \mid X\right)-E\left(\frac{D \psi(P, X, V, \theta)}{f(V \mid X)} \mid V, X\right)\right] .
$$

Efficiency would be obtained in the usual GMM way by having plim $\Omega_{n}=\widetilde{\Sigma}_{0}^{-1}$. In very closely related contexts, Magnac and Maurin (2003) and Jacho-Chavez (2005) show that the estimator that plugs in a nonparametric estimator of $f$ is semiparametrically efficient, being more efficient than estimation using the true $f$ (similar to Hirano, Imbens, and Ridder 2003).

Nonparametric estimation of $f(V \mid X)$ may be problematic in applications where $X$ has moderate or high dimension. In those cases $f$ could be semiparametrically estimated. For example, suppose equation (12) holds but the distribution of $\eta$ is unknown. One could then estimate the parameters of equation (12) by GMM, apply a one dimensional nonparametric density estimator to the estimated residuals $\widehat{\eta}$ from that equation to obtain $\widehat{f_{\eta}}$, then estimate $\widehat{\theta}$ by equations (13) and (15). Lewbel and Schennach (2005) provide root $n$ limiting distribution theory for a numerically simple "sorted data" estimator of this form (one that does not require selection of kernels or bandwidths), using

$$
\frac{1}{\widehat{f}_{\eta}\left(\widehat{\eta}_{i}\right)}=\frac{\widehat{\eta}_{[i+1]}-\widehat{\eta}_{[i-1]}}{2 n}
$$

where $\widehat{\eta}_{[i+1]}$ is the smallest value of $\widehat{\eta}_{1}, \ldots, \widehat{\eta}_{n}$ that is greater than $\widehat{\eta}_{i}$ and $\widehat{\eta}_{[i-1]}$ is the largest value of $\widehat{\eta}_{1}, \ldots, \widehat{\eta}_{n}$ that is smaller than $\widehat{\eta}_{i}$. 


\subsection{Linear Two Stage Least Squares}

Suppose that

$$
P^{*}=Y^{\prime} \beta_{Y}+V \beta_{V}+\varepsilon, \quad E(\varepsilon Z)=0, \quad \varepsilon|V, X \sim \varepsilon| X .
$$

This is just the special case of model (9) where $h\left(P^{*}, Y, V, \beta\right)$ is $P^{*}-Y^{\prime} \beta_{Y}-$ $V \beta_{V}$. As before, some of the elements of $Y$ may be endogenous and hence correlated with $\varepsilon$, so estimation is based on $E(\varepsilon Z)=0$, which for this linear model means that if $P^{*}$ were observed, then the parameters $\beta=\beta_{Y}, \beta_{V}$ could be estimated by regressing $P^{*}$ on $Y, V$ using linear two stage least squares with instruments $Z$.

With this model, the GMM estimator of Theorem 2 and Corollary 3 is based on the moments

$$
E\left[Z W\left(P-Y^{\prime} \beta_{Y}-V \beta_{V}\right)\right]=0
$$

and so simplifies to estimating $\beta$ by linearly regressing $W P$ on $W Y, W V$ using two stage least squares with instruments $Z$. Define

$$
\begin{aligned}
\Delta & =\left[E\left(W\left(\begin{array}{l}
Y \\
V
\end{array}\right) Z^{\prime}\right) E\left(Z Z^{\prime}\right)^{-1} E\left(W Z\left(\begin{array}{l}
Y \\
V
\end{array}\right)\right)\right]^{-1} E\left(W\left(\begin{array}{l}
Y \\
V
\end{array}\right) Z^{\prime}\right) E\left(Z Z^{\prime}\right)^{-1} \\
\beta & =\Delta E(W Z P)
\end{aligned}
$$

The estimator is just these equations, replacing expectations with sample averages.

Suppose $f(V \mid X)$ is parameterized as $f(V \mid X, \lambda)$ and we have some estimator for the vector $\lambda$ satisfying

$$
\sqrt{n}(\widehat{\lambda}-\lambda) \stackrel{d}{\longrightarrow} N\left[0, \operatorname{var}\left(Q_{\lambda}\right)\right]
$$

for some influence function $Q_{\lambda}$. For example, $\lambda$ might consist of means or other moments of $V, X$ and $\widehat{\lambda}$ could be the corresponding sample moments, or $\lambda$ could be estimated by a separate GMM, or by maximum likelihood as before. Then the estimator $\widehat{\beta}$ is a linear two stage least squares regression of $P D / f(V \mid X, \widehat{\lambda})$ on $(Y, W) D / f(V \mid X, \widehat{\lambda})$ using instruments $Z$. With independent, identically distributed observations, standard limiting distribution theory for parametric two step estimation (see, e.g., section 6 of Newey and McFadden 1994) then gives

$$
\sqrt{n}(\widehat{\beta}-\beta) \stackrel{d}{\longrightarrow} N\left[0, \Delta \operatorname{var}\left(Q_{\beta}-W Z Y^{\prime} \beta\right) \Delta^{\prime}\right]
$$


where

$$
Q_{\beta}=W Z P\left(1-Q_{\lambda}^{\prime} \frac{\partial \ln f(V \mid X, \lambda)}{\partial \lambda}\right)
$$

If instead of a parametric density, we use a kernel or other sufficiently regular nonparametric density estimator $\widehat{f}(V \mid X)$, then let $\widehat{W}=D / \widehat{f}(V \mid X)$ and estimate $\beta$ by regressing $\widehat{W} P$ on $\widehat{W} Y, \widehat{W} V$ using linear two stage least squares with instruments $Z$. This is then just a special case of GMM with a nonparametric density estimator as described in the previous section, which yields the same limiting distribution (19) as the parametric density case, except that now

$$
Q_{\beta}=W Z P+E(W Z P \mid X)-E(W Z P \mid V, X) .
$$

These two stage least squares estimators do not require numerical searches, so it would also be computationally feasible to estimate limiting distributions by bootstrapping.

\subsection{A Numerically Trivial Estimator}

Consider the weighted least squares model with a semiparametric specification of $f$, specifically,

$$
\begin{aligned}
P^{*} & =Y^{\prime} \beta_{Y}+V \beta_{V}+\varepsilon, \quad E(\varepsilon Z)=0, \\
V & =X^{\prime} \alpha+\eta, \quad \eta \perp \varepsilon, X
\end{aligned}
$$

and $D$ given by equation (1). Assume the distributions of errors $\varepsilon, \eta$ and unobservables $A^{*}, M^{*}$ are unknown. This model is a special case of equations (17) and (12), and provides a compromise between parametric vs nonparametric $V$ density estimation. A numerically trivial estimator (which combines estimators described in the previous sections) consists of the following steps.

First estimate $a$ by linearly regressing $V$ on $X$ using ordinary least squares, and then let $\widehat{\eta}_{i}=V_{i}-X_{i}^{\prime} \widehat{\alpha}$ at each data point $i$. By equation (13) in this model we have $W=D / f_{\eta}(\eta)$, so by equation (16) at each data point $i$ we may construct $\widehat{W}_{i}$ using

$$
\widehat{W}_{i}=\frac{\widehat{\eta}_{[i+1]}-\widehat{\eta}_{[i-1]}}{2 n} D_{i}
$$

where $\widehat{\eta}_{[i+1]}$ is the smallest value of $\widehat{\eta}_{1}, \ldots, \widehat{\eta}_{n}$ that is greater than $\widehat{\eta}_{i}$ and $\widehat{\eta}_{[i-1]}$ is the largest value of $\widehat{\eta}_{1}, \ldots, \widehat{\eta}_{n}$ that is smaller than $\widehat{\eta}_{i}$. To handle the endpoints, for $\widehat{\eta}_{j}=\min \left(\widehat{\eta}_{1}, \ldots, \widehat{\eta}_{n}\right)$ let $\widehat{\eta}_{[j-1]}=\widehat{\eta}_{j}$ and for $\widehat{\eta}_{j}=\max \left(\widehat{\eta}_{1}, \ldots, \widehat{\eta}_{n}\right)$ let $\widehat{\eta}_{[j+1]}=\widehat{\eta}_{j}$. 
Alternatively, instead of equation (20), $f_{\eta}\left(\eta_{i}\right)$ could be estimated using a one dimensional nonparametric kernel density estimator. Finally, estimate $\beta$ by linearly regressing $\widehat{W} P$ on $\widehat{W} Y, \widehat{W} V$ using two stage least squares with instruments $Z$. Lewbel and Schennach (2005) provides relevant root $n$ asymptotic distribution theory. They also show that no asymptotic trimming is required, and that semiparametric efficiency can be obtained using $\widehat{\eta}_{[i+k]}-\widehat{\eta}_{[i-k]}$ where $k \rightarrow \infty$ at an arbitrarily slow rate.

This estimator consists only of sorting data and some linear regressions, so bootstrapping by repeatedly resampling from the original data with replacement would be computationally trivial. Consistency of the bootstrap may require $k \rightarrow$ $\infty$. Estimation in the later empirical application keeps $k=1$.

\subsection{Outliers and Trimming}

The estimators entail division by a density $f(V \mid X)$. This raises potential numerical issues when the density is close to zero. Formally, asymptotic trimming is generally required for high dimensional nonparametric density plug ins to avoid boundary bias issues, and while not required in theory for root $n$ convergence with a parametric $\widehat{f}$, may still be advisable in terms of mean squared error. Essentially, the issue is that $f\left(V_{i} \mid X_{i}\right)$ and hence $W_{i}$ will be extremely small, making $U_{i}$ possibly extremely large, for observations $i$ where $V_{i}$ is moderately large, and such observations may dominate the associated sample averages. Basically, such observations $i$ will be outliers. This suggests that one should look for and possibly discard outliers in the moments $U_{i}$ or two stage least squares errors. One could more formally replace the weighted means in the GMM or two stage least squares with robust estimators of such means, e.g., discarding some small percentage of the observations in the regressions that have the largest residuals.

\section{A Factory Investment Model}

Let $P_{i}$ be the rate of investment in manufacturing plant $i$, defined as the level of investment in a year divided by the beginning of the year value of the plant's capital, and let $Q_{i}$ be Tobin's Q for the plant. Classical models of firm behavior (e.g., Eisner and Strotz 1963) imply $P_{i}$ proportional to $Q_{i}$, where the constant of proportionality is inversely related to the magnitude of adjustment costs. However, simple estimates of this relationship at varying levels of aggregation typically find 
a very low constant of proportionality (see, e.g., Summers 1981 or Hayashi 1982), implying implausibly large adjustment costs.

Another empirical finding inconsistent with proportionality is that plant or firm level data on investment show many periods of zero or near zero investment, alternating with periods of high investment. See, e.g., Doms and Dunne (1998) and Nilsen and Schiantarelli (2003). These empirical findings are generally attributed to discontinuous costs of adjustment, due to factors such as irreversibility or indivisibility of investments. See Blundell, Bond, and Meghir (1996) for a survey.

One difficulty in applying $Q$ models to disaggregate data is that accurate measures of an appropriate firm or plant level marginal $Q$ are difficult to construct. Typical proxies for $Q$ are sales or profit rates. Let $R_{i}$ be the profit rate of plant $i$, defined as profits derived from the plant in a year divided by the beginning of the year capital. A problem with the use of a proxy like $R_{i}$ is that it may be endogenous, since profits depend on the level of investment.

Let $C_{i}$ be the cost of investment in plant $i$ in a year, divided by capital at the beginning of the year. Based on the model of Abel and Eberly (1994), assume plant $i$ has investment costs of the form

$$
C_{i}=a_{1 i} I\left(P_{i} \neq 0\right)+a_{2 i} P_{i}+a_{3} P_{i}^{2}
$$

The term $a_{1 i}$ is plant $i$ 's fixed (per unit of capital) cost associated with any nonzero investment, $a_{2 i}$ is the price of investment, which can vary across plants, and $a_{3}$ is a quadratic adjustment cost parameter. Following the derivations in Abel and Eberly (1994), given the above investment cost function the firm chooses investment $P_{i}$ to maximize the present value of current and expected future profits, resulting in a model of the form

$$
\begin{aligned}
P_{i} & =\left[g^{*}\left(a_{2 i}\right)+\beta_{1}^{*} Q_{i}\right] D_{i} \\
D_{i} & =I\left[Q_{i}>g\left(a_{1 i}, a_{2 i}\right)\right]
\end{aligned}
$$

Where the functions $g^{*}$ and $g$ and the parameter $\beta_{1}^{*}$ depend on features of the firm's intertemporal profit function. Abel and Eberly's model also implies disinvestment $\left(P_{i}<0\right)$ if $Q_{i}$ is below some lower bound. Very few firms in the data set have negative investment, so that outcome will not be explicitly modeled. The above equations for $P$ and $D$ hold as written for all firms if $P_{i}$ is set to zero for any firm having negative investment.

The above equations show that profit maximization yields a model that has a sample selection structure. It also has the features that the outcome $P$ is linear 
in $Q$ when $D=1$, and that the fixed cost parameter $a_{1 i}$ appears only in the expression for $D$.

Comparing this model for $D$ with equation (1) shows that $A^{*}$ will be infinite, but one could construct more elaborate versions that would give rise to a finite upper bound, e.g., if it were the case that a firm would build a new plant rather than invest in the old one when the benefits from investment were sufficiently large.

Marginal plant level Tobin's Q is not observed, and is proxied by the profit rate $R_{i}$. Specifically, $Q_{i}$ is assumed to be linear in $R_{i}, X_{2 i}$, and an additive error, where $X_{2 i}$ is a vector of observable attributes of the firm or plant. The function $g^{*}\left(a_{2 i}\right)$ is also assumed to be linear in $X_{2 i}$ and an additive error. This yields the outcome model $P_{i}=\left(R_{i} \beta_{1}+X_{2 i}^{\prime} \beta_{2}+\varepsilon_{i}\right) D_{i}$. The error term $\varepsilon_{i}$ will be independent of profits, or nearly so, if a collection of restrictive assumptions hold (including constant returns to scale, competitive product markets, and a first order autoregressive model for $R_{i}$. See Abel and Eberly 1994 for details). Because these assumptions are unlikely to hold in practice, the estimator here will not require $\varepsilon_{i}$ to be independent of the profit rate $R_{i}$, and so will allow for possible endogeneity of profits.

Let $Z_{i}$ be a vector of instruments, comprised of $Z_{1 i}$ defined as the lagged profit rate, and plant characteristics $Z_{2 i}=X_{2 i}$. Define the function $H$ by $H(z)=$ $E(R \mid Z=z)$, and define $\varepsilon_{R i}$ by $R_{i}=H\left(Z_{i}\right)+\varepsilon_{R i}$. The function $H$ is unknown. Because of endogeneity of profits, the error term $\varepsilon_{R i}$ may be correlated with $\varepsilon_{i}$, and is not assumed to be independent of $Z_{i}$.

Let $V_{i}$ be a measure of the size of plant $i$. In standard $Q$ models, the relationship of the investment rate $P$ to $Q$ does not depend on the size of the firm or plant, except to the extent that both $P$ and $Q$ are expressed in "per unit of capital" terms. However, in empirical applications it is generally found that size does matter. The Abel and Eberly model provides one possible explanation, by allowing $V_{i}$ to affect the fixed cost of investments $a_{1 i}$. In particular, $a_{1 i}$ is the fixed cost per unit of capital, so if true fixed costs (in absolute terms) are present, then $a_{1 i}$ will be a decreasing function of $V_{i}$. Nilsen and Schiantarelli (2003) find strong statistical evidence of this relationship, including much greater incidences of zero investments in small versus large plants. They attribute this relevance of plant size both to the presence of absolute as well as relative fixed costs and to potential indivisibilities in investment. Other studies confirm the relevance of size on the decision to invest, but most cannot separate plant level effects from other factors, because they use more aggregated firm or industry level data. Since $V_{i}$ is a plant characteristic, it may also appear everywhere in that model that $X_{2 i}$ appears. 
Based on the above, it is assumed that $a_{1 i}$ depends on $V_{i}$ and may also depend on other characteristics of the plant, firm, or industry, both observed $X_{2 i}$ and unobserved $e_{i}$. Consistent with the presence of absolute fixed costs, Nilsen and Schiantarelli (2003) find that $D$ is monotonically increasing in $V$, so (recalling Lemma 1) we may write the resulting selection equation as $D_{i}=I[0 \leq$ $\left.V_{i}+M\left(R_{i}, X_{2 i}, e_{i}\right)\right]$ for some function $M$, where $e_{i}$ denotes a vector of unobserved variables or errors that affect the decision to invest. The unobservables $e_{i}$ will in general be correlated with the other unobservables in the system, $\varepsilon_{i}$ and $\varepsilon_{R i}$. Also, in the Abel and Eberly model the function $g$ is nonlinear in $a_{1 i}$ (it's related to a root of a quadratic equation) and $a_{1 i}$ itself is an unknown, possibly nonlinear function of $V_{i}$. Therefore $M$, which is based on $g, a_{1 i}$, and $a_{2 i}$, is an unknown function that is likely to be nonlinear.

The above derivations yield the following system of equations

$$
\begin{aligned}
P_{i} & =\left(R_{i} \beta_{1}+X_{2 i}^{\prime} \beta_{2}+V_{i} \beta_{3}+\varepsilon_{i}\right) D_{i} \\
D_{i} & =I\left[0 \leq V_{i}+M\left(R_{i}, X_{2 i}, e_{i}\right)\right] \\
R_{i} & =H\left(Z_{i}\right)+\varepsilon_{R i}
\end{aligned}
$$

The profit rate $R_{i}$ is endogenous, correlated with $\varepsilon_{i}$, and the selection unobservables vector $e_{i}$ is correlated with both the investment rate error $\varepsilon_{i}$ and the profit rate error $\varepsilon_{R i}$. The joint distribution of these errors, and the functions $M$ and $H$, are unknown. The goal is estimation of the parameters $\beta$. The coefficient of $R_{i}$, which is $\beta_{1}$, is of particular interest as the proxy for the relationship between investment and $Q$.

Equation (21) takes the form $P=P^{*} D$ with $P^{*}$ linear, so this paper's two stage least squares estimators will be used. In the notation of the previous sections of this paper, $P, D, V$, and $Z$ are the same, $Y$ is $R, X_{2}$, and $X$ is the union of the elements of $R, X_{2}$, and $Z$.

\subsection{Data and Estimation}

The model is estimated using data from Norwegian manufacturing plants in 1986, ISIC codes (industry numbers) 300-390. The available sample consists of $n=974$ plants. See Nilsen and Schiantarelli (2000) for a full data description. The main advantage over more conventional investment data sets is that data here are available at the level of individual manufacturing plants, rather than firm level data that is aggregated across plants. This is important because the theory involving fixed 
costs applies at the plant level, and averaging this nonlinear model across plants or firms may introduce aggregation biases, particularly in the role of variables affecting $D_{i}$, such as $V_{i}$.

$P_{i}$ is investment just in equipment in plant $i$ in 1986, divided by the beginning of the year's capital stock in the plant. The investment rate $P_{i}$ equals zero in about tweny per cent of the plants. Around two percent of plants have negative investment. Consistent with the model, negative investment plants have $P_{i}$ set to zero. The selection function is then $D_{i}=I\left(P_{i}>0\right)$.

The variable $R_{i}$ is profits attributable to plant $i$ in 1986, divided by the beginning of the year's capital stock. Plant characteristics $X_{2 i}$ consist of a constant term, dummy variables for two digit ISIC code, and dummies indicating whether the firm is a single plant or multiplant firm, and if multiplant, whether plant $i$ is the primary manufacturing facility or a secondary plant. The instruments $Z_{i}$ are comprised of $Z_{2 i}=X_{2 i}$, and $Z_{1 i}$ defined as lagged $R_{i}$, so $Z_{1 i}$ is the profit rate for the plant in 1985. The size variable $V_{i}$ is taken to be the log of employment at plant $i$ in an earlier year. Experiments with other measures of size, such as lagged capital stock, yielded similar results.

To apply this paper's estimator for $\beta$, we need the assumptions of Corollary 3 to hold. The structural model is equations (21), (22), and (23). This requires that the unobservables in the model, $e, \varepsilon$, and $\varepsilon_{R}$, be conditionally independent of $V$, conditioning on $Z$. The most likely source of violation of this assumption is from the replacement of $Q$ with $R$ (in part because $Q$ should subsume all dynamic effects, but $R$ need not). This was also the motivation for inclusion of the term $\beta_{3} V_{i}$ in equation (21).

The required support conditions for $V$ imply that, at any given time, some plants could be so small that they will not invest regardless of their values for $X_{2}$ and $e$, while other plants could be so large that they definitely invest. Over time plant sizes change, both through the model via investment, and by depreciation, closings, technology change, etc., so the model does not require the existence of plants that are permanently static or permanently growing.

Empirically, the supports of the continuous variables in this model are unknown, so the required support conditions cannot be directly verified. However, indirect evidence is favorable. In this data set $V$ takes on a large range of values relative to the other covariates. For example, as a measure of data spread, the standard deviation of $V$ is 1.16, while the profit rate $R$ has a standard deviation of .17. In the applications where, for comparison, the selection equation is parameterized, the systematic component of $M^{*}$, modeled as $X_{2}^{\prime} \gamma$, has a standard deviation comparable to that of $V$, ranging from .80 to 1.40 depending on the model and the 
estimator. In a Monte Carlo analysis, Lewbel (2000) found that the similar binary choice estimator generally performed well when the standard deviation of $V$ was comparable in magnitude to the standard deviation of $M^{*}$.

Very strong alternative assumptions are required to estimate $\beta$ by other means, such as maximum likelihood. The model can be rewritten as

$$
\begin{aligned}
R_{i} & =H\left(Z_{i}\right)+\varepsilon_{R i} \\
P_{i} & =\left[H\left(Z_{i}\right) \beta_{1}+X_{2 i}^{\prime} \beta_{2}+V_{i} \beta_{3}+\left(\varepsilon_{R i} \beta_{1}+\varepsilon_{i}\right)\right] D_{i} \\
D_{i} & =I\left[0 \leq V_{i}+M\left(H\left(Z_{i}\right)+\varepsilon_{R i}, X_{2 i}, e_{i}\right)\right]
\end{aligned}
$$

The parametric model that will be estimated for comparison is

$$
\begin{aligned}
R_{i} & =Z_{i}^{\prime} b+\widetilde{\varepsilon}_{R i} \\
P_{i} & =\left[\left(Z_{i}^{\prime} b\right) \beta_{1}+X_{2 i}^{\prime} \beta_{2}+V_{i} \beta_{3}+\widetilde{\varepsilon}_{i}\right) D_{i} \\
D_{i} & =I\left[0 \leq V_{i}+\left(Z_{i}^{\prime} b\right) \gamma_{1}+X_{2 i}^{\prime} \gamma_{2}+\widetilde{e}_{i}\right]
\end{aligned}
$$

where the errors $\left(\widetilde{e}_{R i}, \widetilde{\varepsilon}_{i}, \widetilde{e}_{i}\right)$ are assumed to be trivariate normal and independent of $Z_{i}$ and $V_{i}$. Unlike the general semiparametric specification, this parametric model requires the simultaneous estimation of the equations for $R_{i}$ and $D_{i}$ along with the $P_{i}$ equation. The parametric model also assumes that the functions $H$ and $M$ are linear, that the errors and unobservables $\varepsilon_{R i}$ and $e_{i}$ can be subsumed into a single additive error $\widetilde{e}_{i}$, and that the errors are jointly normal and independent of $Z$. Assumptions like these are required for estimation of the model by any standard method such as maximum likelihood, although they are not well motivated in terms of the economics of the problem. For example, linearity of the function $M$ with a scalar error is inconsistent with the theoretical derivation of the model. This illustrates the value of the semiparametric estimation, which does not require such assumptions.

\subsection{Empirical Results}

Table 1 summarizes results for six different estimators. For brevity, Table 1 only reports estimates of the coefficient of interest, $\beta_{1}$. A complete list of all parameter estimates in all equations, along with the Gauss code used to generate them, are available from the author on request.

Let $X_{1 i}=R_{i}, X_{2 i}$ and let $\beta$ denote the corresponding coeficients in equation (21). The first and second estimators in Table 1, labeled OLS and TSLS, ignore the sample selection problem, and just estimate the equation $P_{i}=X_{1 i}^{\prime} \beta+\widetilde{\varepsilon}_{i}$ by 
ordinary least squares and two stage least squares, respectively (the latter using instruments $Z_{i}$ ).

The third estimator, labeled Heckman, controls for sample selection parametrically, but does not control for possible endogeneity. This is the two equation parametric model $P_{i}=\left(X_{1 i}^{\prime} \beta+\widetilde{\varepsilon}_{i}\right) D_{i}$ and $D_{i}=I\left[0 \leq V_{i}+X_{1 i}^{\prime} \gamma+\widetilde{e}_{i}\right]$, assuming $\widetilde{\varepsilon}_{i}$ and $\widetilde{e}_{i}$ are jointly normal and independent of $V_{i}$ and $X_{1 i}$. This third estimator is the standard Heckman model, estimated using maximum likelihood.

The fourth estimator, labeled Endogenous ML, is maximum likelihood estimation of the entire three equation parametric model (25), which entails simultaneously estimating the parametric selection, outcome, and instrument equations, assuming $\widetilde{e}_{R i}, \widetilde{\varepsilon}_{i}$, and $\widetilde{e}_{i}$ are jointly normal and independent of $Z_{i}$ and $V_{i}$.

The remaining estimators are this paper's estimators from section 4.4. The fifth estimator, Weighted OLS, is a linear least squares regression of $\widehat{W}_{i} P_{i}$ on $\widehat{W}_{i} X_{1 i}$, where the weights are $\widehat{W}_{i}=D_{i} / \hat{f}\left(V_{i} \mid X_{1 i}\right)$. This semiparametrically controls for selection but not for endogeneity, and so corresponds to estimating $\beta$ when the true model is defined by the system of two equations (21) and (22), assuming $\beta_{3}=0$ (see below for more on this point) and $\varepsilon_{i}$ is uncorrelated with $X_{1 i}$.

The final estimator, Weighted TSLS, is a linear two stage least squares regression of $\widehat{W}_{i} P_{i}$ on $\widehat{W}_{i} X_{1 i}$ using instruments $Z_{1 i}$, where the weights are $\widehat{W}_{i}=$ $D_{i} / \hat{f}\left(V_{i} \mid X_{i}\right)$ with $X_{i}=X_{1 i}, Z_{1 i}$. This estimator semiparametrically controls for both selection and endogeneity, and so corresponds to estimating $\beta$ when the true model is defined by the general structure of equations (21), (23), and (22).

A kernel density estimator is used to construct $\hat{f}\left(V_{i} \mid X_{i}\right)$. A quartic kernel is used for continuous regressors, calculated for each cell of the discrete regressors and averaged across cells (examples of estimation using the more trivial parametric and ordered data density estimators are provided later). The kernel bandwidth is chosen by ordinary cross validation. No density trimming was used. Estimates were also generated with bandwidth's constructed using the procedure described in Lewbel (2000), and by halving the cross validated bandwidths to undersmooth as required for root $n$ convergence. Those are not reported, since the resulting coefficient estimates were not very sensitive to bandwidth choice.

The semiparametric estimators are computationally quick and straightforward, since they only entail kernel density estimation and linear two stage least squares. In contrast, the maximum likelihood estimates were quite difficult to obtain, with frequent numerical problems and failures to converge. Attempts to replicate the analysis for a different year of data failed because no converged values for the maximum likelihood estimator could be obtained. The difficulty with maximum 
likelihood is that some parameters are intrinsically difficult to identify in the sense that the likelihood function is relatively flat in directions that involve changing these parameters. These parameters include correlations between the latent selection error $\widetilde{e}_{i}$ and the other model errors, and many structural parameters were sensitive to the estimates of these correlations. The semiparametric estimator does not require estimation of these difficult to obtain nuisance parameters.

Table 1 reports estimates imposing $\beta_{3}=0$ in equation (21). This is a necessary assumption for the weighted OLS estimator (because $U$, which equals $\varepsilon$ times instruments, must be conditionally independent of $V$, and in weighted OLS the instruments are the regressors, which include $V$ when $\beta_{3}$ is nonzero). The other estimators do not require this assumption in theory, however, the Endogenous ML estimates failed to converge when $\beta_{3}$ was allowed to be nonzero, and when the other estimators were redone allowing $\beta_{3}$ to be nonzero, the resulting estimates of $\beta_{3}$ were tiny and completely insignificant statistically. Also, imposing $\beta_{3}=0$ only slightly changed the resulting estimates of $\beta_{1}$. Note that having $\beta_{3}=0$ is consistent with a model where $V_{i}$ only affects fixed costs of investment.

In both the parametric and semiparametric models, controlling for selection and for endogeneity each raises the estimate of $\beta_{1}$ (recall the empirical finding in this literature is that naive estimates of this coefficient are implausibly low). The semiparametric estimates are comparable to, though generally higher than, the corresponding parametric model estimates.

One could easily question whether $V$ satisfies all of the required assumptions in this application. Of course the maximum likelihood estimators also require some rather suspect, though very different, strong assumptions. Still, the empirical results are sensible, suggesting at a minimum that the semiparametric estimator produces plausible results here. Moreover, the similarity in estimates obtained by the parametric and semiparametric estimators should increase confidence in at least rough validity of the underlying model.

\subsection{Monte Carlo Simulation}

To assess the performance of the proposed estimator, a Monte Carlo simulation based on the investment model application is provided. For the simulation, the true model is taken to be the three equation parametric model (25), without plant or industry dummies, and $\beta_{3}=0$. Parameter values are taken to equal the estimated coefficients from applying maximum likelihood to the investment data (the Endogenous ML model in Table 1) with the full set of plant and industry dummies included in $X_{2 i}$. The intercept term in the outcome equation is then taken to equal 
the mean in the real data of $X_{2 i}^{\prime} \beta_{2}$, and the intercepts for the other two equations are defined analogously. The exogenous variables $V$ and $Z$, corresponding to the size and lagged profit rate variables, are drawn as independent normals with means and variances matching those in the data. The covariance matrix of the normal model errors $\left(\widetilde{e}_{R i}, \widetilde{\varepsilon}_{i}, \widetilde{e}_{i}\right)$ is then constructed so that the means, variances, and covariances of the endogenous variables $R_{i}, P_{i}$, and $D_{i}$ generated by the parametric model match those in the real data. The sample size is the same as the real data, 974 observations.

Simulated data were drawn in this way five thousand times, and each of the six estimators described in Table 1 were applied to each replication. With each replication, the same code that was used on the real data was applied to the simulated data to provide estimates of both the coefficients and the standard errors.

Table 2 reports summary statistics on the distribution of the estimated profit coefficient in the outcome equation from these simulations. Corresponding summary statistics on all of the estimated parameters, along with the Gauss code used to generate them, is available from the author on request. Reported summary statistics include moments, quantiles, root mean squared errors, and mean and median absolute errors. Also reported is the mean across replications of the estimated standard errors, and the fraction of simulations in which the true coefficient was within two estimated standard errors of the estimated coefficient.

By the Monte Carlo design, the endogenous ML estimator is consistent and efficient, and so provides an asymptotically best case benchmark. The results show that this ML estimator is mean and median unbiased, with a smaller root mean square error than the other estimators, as expected. One way in which ML behaved poorly was that its estimated standard errors were much too large, providing $100 \%$ coverage of what is supposed to be a $95 \%$ confidence interval. This illustrates the problem noted in the real data analysis that the ML estimates are sensitive to the estimated covariance matrix of the model errors, which in turn is estimated imprecisely because one of the errors is latent. Equivalently, in this application ML is a highly nonlinear function, making the linearization required for standard error estimation a poor approximation.

The OLS, TSLS, Heckman, and Weighted OLS estimators are inconsistent for this design. The simulated estimates of each of these estimators show considerable bias, with means and medians that are very similar to estimates reported with real data (compare the next to last column of Table 1 with the mean and median columns of Table 2). The estimated standard errors of these estimators also closely match the real data estimated standard errors. The Weighted OLS estimator delivers estimates close to those of the Heckman model, as it should. 
The Weighted TSLS estimator has about a ten percent mean and median bias, and a standard deviation about double that of ML. This is the price paid for the generality of the semiparametric estimator. Unlike ML, the standard errors of the weighted TSLS are quite accurate, resulting in 96 percent.of coverage for what is supposed to be a 95\% confidence interval. This paper's proposed weighted TSLS estimator does not require estimation of the latent errors (indeed, it does not involve any estimation at all of the selection equation), which may explain its better behavior regarding standard error estimation.

In this Monte Carlo design all of the variables and errors, including $V$, have unbounded support and no asymptotic trimming was applied. These estimates can be consistent based on Theorem 1, but formally the unbounded support violates our root $n$ limiting distribution theory (since in these designs $A^{*}$ is infinite). Nevertheless, the Monte Carlo results suggest that the root $n$ limiting distribution theory (with a small limiting bias) provides a good approximation to the observed sampling distribution. Also, these results show that the finite sample bias from the proposed estimator is much smaller than that of other simple biased estimators which ignore either selection or endogeneity. Some experiments with asymptotic trimming were performed, but they are not reported because they did not produce any improvements in the simulations.

\section{Wages and Schooling}

This section describes an empirical application in which $A^{*}$ is finite. Let $-V_{i}$ be the $\log$ cost of a year of school, and let $M_{i}^{*}$ denote an individual $i$ 's unobserved utility from education (comparably normalized), so the larger $M_{i}^{*}+V_{i}$ is, the more education individual $i$ will choose to obtain. Let $D_{i}$ equal one if $i$ has an undergraduate degree and no post graduate education, and zero otherwise. Then $D_{i}=I\left(0 \leq M_{i}^{*}+V_{i} \leq A_{i}^{*}\right)$, where $i$ does not get an undergraduate degree if $M_{i}^{*}+V_{i}<0$ and gets some graduate education if $M_{i}^{*}+V_{i} \geq A_{i}^{*}$. This simple model of the selection equation ignores dynamic optimization issues in schooling choice, but does allow thresholds to vary either randomly or systematically across individuals (see, e.g., Cameron and Heckman 1998 or Carneiro, Hansen, and Heckman 2003), and leaves unspecified the many observables and unobservables that affect utility and thresholds, that is, $M_{i}^{*}$ and $A_{i}^{*}$.

Let the potential outcome $P_{i}^{*}=Y_{i}^{\prime} \beta+\varepsilon_{i}$ be the log wages individual $i$ would get if he or she chose to obtain an undergraduate degree but no graduate education, where $Y_{i}$ is a vector of observed covariates and $E\left(Y_{i} \varepsilon_{i}\right)=0$, so we do 
not have endogenous regressors in this example. The goal is estimation of $\beta$ and hence the effect on wages from obtaining an undergraduate degree. The selection problem is that we can only observe $P_{i}^{*}$ for individuals having $D_{i}=1$, and we can expect $M_{i}^{*}$ and possibly also $A_{i}^{*}$ to correlate with $P_{i}^{*}$ in unknown ways. We may therefore directly apply the estimators described in section 4.4, to obtain $\widehat{\beta}$ by a linear ordinary least squares regression of $\widehat{W}_{i} P_{i}$ on $\widehat{W}_{i} Y_{i}$, where $P_{i}$ is individual $i$ 's observed $\log$ wage and $\widehat{W}_{i}$ is $D_{i}$ divided by an estimate of the conditional density of $V_{i}$ given $X_{i}$. This density is estimated three ways. The first uses the same nonparametric estimator as in the investment application. The second assumes $V_{i}=X_{i}^{\prime} \alpha+\eta_{i}$ where $\eta_{i}$ is an independent normal error, with the $V$ and $W P$ equations estimated jointly by GMM. The third is the numerically trivial estimator of section 4.5, which again assumes $V_{i}=X_{i}^{\prime} \alpha+\eta_{i}$, but now with the independent error $\eta_{i}$ having an unknown density that is nonparametrically estimated using Lewbel and Schennach (2005). This last estimator is sequential, where first $V$ is linearly regressed on $X$, then the errors in that regression are sorted and differenced to construct $\widehat{W}$ using equation (20), and last $\beta$ is estimated by a linear least squares regression of $\widehat{W}_{i} P_{i}$ on $\widehat{W}_{i} Y_{i}$.

For comparison, estimates are also obtained by just regressing $P_{i}$ on $Y_{i}$ for those individuals having $D_{i}=1$. This regression suffers from selection bias, unless the $D_{i}=0$ observations are missing at random. Also reported are maximum likelihood estimates of a two equation system where $A_{i}^{*}$ is a constant and $M_{i}^{*}$ is modeled as $X_{i}^{\prime} \gamma+e_{i}$ and assuming $e_{i}, \varepsilon_{i}$ are bivariate normal, independent of $Y_{i}$ and $X_{i}$. The results are all in Table 3 .

The data set used here, and the choice of regressors $Y_{i}, X_{i}$, and $V_{i}$, is from Chen (2003), constructed primarily from the National Longitudinal Survey for Youth (NLSY). $V_{i}$ is minus the log of the total expense of attending a local in state public college, deflated by the local average hourly wage of unskilled workers that prevailed when $i$ was 17 years old. Alternative choices for $V_{i}$ such as distance to schools as in Card (1995) could be used, but did not vary as much as this cost measure. $X_{i}$ consists of a constant term, a scholastic ability index (constructed as a composite of test scores), dummy variable indicators for a parent that went to college, whether $i$ is black, whether $i$ is male, and whether $i$ 's cohort is from the 1980 's or the 1990's. $Y_{i}$ equals $X_{i}$ plus additional dummies indicating one to five years of work experience and over five years of work experience. The total sample size is 7013 individuals, with 3775 of them having $D_{i}=1$. See Chen (2003) and Chen and Khan (2003) for more details on the construction and use of this data set, and Kane and Rouse (1995) for related results on NLSY data.

The estimates from all the estimators are roughly comparable, which shows 
that the density weighted estimators are at least not generating wild estimates. A possible exception is the normal weighted OLS, which has a few implausible coefficient estimates, such as a negative effect on having over five years of work experience. Normality may not be a reasonable assumption for $\eta_{i}$, or may be resulting in some extreme outliers that should be trimmed out. It is notable that the numerically trivial estimator (sorted weighted OLS in Table 3) appears to work well.

One substantial difference across the estimates is that OLS gives a significant 5 percent increase in wages resulting from a parent having a college education, while MLE gives an implausible negative 5 percent effect. The semiparametric kernel and sorted density estimates are near zero and completely insignificant statistically (unlike every other coefficient, the sign of this coefficient in the kernel estimator changes when a different bandwidth is used). Selection bias may cause the OLS estimate to be too high, because parent's education is a strong determinant of whether the child goes to college.

Another notable (though not statistically significant) difference is that all the semiparametric estimators say the increase from the 1980's to the 1990's in real wages from having a degree, after controlling for other covariates, is around 12 percent or more, while the MLE and OLS give gains of only 9 and 10 percent. The semiparametric estimates also have higher scholastic test score effects on wages than MLE (though not as high as OLS).

Most of the OLS estimates are not very different from the others, which suggests that in this application the effects of selection bias may not be very large. It may be the case that, with two sided censoring, the selection bias due to censoring from above partially offset the selection bias due to censoring from below.

Empirical and theoretical objections have been raised regarding the validity and exogeneity of access to schooling measures like distance and average schooling costs (see, e.g., Carneiro and Heckman 2002, Carneiro, Hansen, and Heckman 2003, and Hogan and Rigobon 2003), so the results reported here must all be interpreted with caution, particularly if the resulting estimates are to be interpreted as measures of the returns to schooling.

Similar models could be estimated for other amounts of schooling. Another caveat on interpreting these results is that only employed individuals are included in the data set, so the results are conditional on finding employment. The estimators in this paper could also be used to estimate the differences in probabilities of employment resulting from schooling, by defining $P_{i}$ to be an indicator of employment and estimating a nonlinear or nonparametric discrete choice model, again controlling for selection by $V$ density weighting. 


\section{Conclusions}

Instead of weighting by a propensity score, this paper shows that selection can be addressed through weighting by the conditional density of one covariate $V$. Strong support and independence assumptions about $V$ replace the usual strong assumptions about the joint distribution of unobservables affecting selection or treatment and outcomes. Essentially, this density weighting converts expectations of data censored by $D$ into expectations of uncensored data. As a result, selection problems can be handled in conjunction with any estimator that is based on expectations. This paper focused on GMM type estimators, including least squares, instrumental variables, and maximum likelihood, but the method could also be used with other estimators based on expectations. For example, Theorem 1 and its corollaries can be extended to identify and estimate $E\left(U^{*} \mid X\right)$ (assuming $A^{*} \perp X$ ), essentially by replacing the numerator and denominator of equation (5) with nonparametric regressions of $U W$ on $X$ and of $W$ on $X$, respectively. Another example is estimation of panel models with fixed effects and selection. If $P_{i t}^{*}=Y_{i t}^{\prime} \beta+\alpha_{i}+\varepsilon_{i t}$ and $P_{i t}=P_{i t}^{*} D_{i}$ then $\beta$ can be estimated by regressing $P_{i t}-P_{i t-1}$ on $Y_{i t-1}-Y_{i t}$ with weights $W_{i}$, thereby differencing out the fixed effects despite the selection problem.

The usefulness of these results in any application of course depends on whether an appropriate covariate $V$ exists. This paper provided two empirical applications, one with $A^{*}$ finite, and the other with the more common case of infinite $A^{*}$. It seems likely that, in at least some applications, one would be more comfortable making strong assumptions about a single observed covariate than the alternative, which requires strong assumptions regarding the joint distribution of all the unobservables that affect both selection and outcomes. If nothing else, one would have more confidence in the results produced by more conventional estimators if the very different identifying assumptions employed here yield comparable estimates.

If more than one plausible candidate for $V$ is present, they could in general be combined. For example, if $D=I\left(0 \leq M^{*}+b_{1} V_{1}+b_{2} V_{2}\right)$, then we could let $V=\widehat{b}_{1} V_{1}+\widehat{b}_{2} V_{2}$ using some consistent (up to scale) estimators for $\widehat{b}_{1}$ and $\widehat{b}_{2}$ such as Powell, Stock, and Stoker's (1989) weighted average derivatives. Alternatively, with GMM estimation we could write one set of moments for estimating $\theta$ using $V_{1}$ as $V$, and a second set of moments for estimating $\theta$ using $V_{2}$ as $V$, and then estimate a single GMM with both sets of moments simultaneously to efficiently combine the information in both sets (though in this case the relative supports of $V_{1}$ and $V_{2}$ are an issue). 
Magnac and Maurin (2003) showed that, for the related binary choice estimator in Lewbel (2000), the large support assumption for $V$ could be relaxed by adding an error tail symmetry assumption, and that the two assumptions (large support vs tail symmetry) are observationally equivalent. As discussed earlier, many semiparametric estimators require a regressor to have a large or infinite support, but it would still be desirable to search for alternatives that could relax the large support requirement in the present sample selection context.

\section{Appendix: Proofs}

Proof of Lemma 1: Define $\pi(V, X)=\operatorname{pr}(D=1 \mid V, X)$. Let $e$ have uniform distribution on $[0,1]$, independent of $V, X$. Define $M^{*}=\pi^{-1}(e, X)$ and $\widetilde{D}=$ $I\left(0 \leq M^{*}+V\right)$. Then

$$
\begin{aligned}
\operatorname{pr}(\widetilde{D} & \left.=1 \mid V, X)=\operatorname{pr}\left[\pi^{-1}(e, X)\right] \leq V \mid V, X\right] \\
& =\operatorname{pr}[e \leq \pi(V, X) \mid V, X]=\pi(V, X)
\end{aligned}
$$

Proof of Theorem 1. First consider the case where $E\left(A^{*}\right)$ is finite. Then

$$
\begin{aligned}
E(U W) & =E\left(\frac{D U^{*}}{f(V \mid X)}\right) \\
& =E\left[E\left(\frac{I\left(0 \leq M^{*}+V \leq A^{*}\right) U^{*}}{f(V \mid X)} \mid X, U^{*}, M^{*}, A^{*}\right)\right] \\
& =E\left[\int_{\operatorname{supp}\left(V \mid X, U^{*}, M^{*}, A^{*}\right)} \frac{I\left(0 \leq M^{*}+v \leq A^{*}\right) U^{*}}{f(V \mid X)} f\left(V \mid X, U^{*}, M^{*}, A^{*}\right) d v\right] \\
& =E\left[\int_{\operatorname{supp}(V \mid X)} I\left(-M^{*} \leq v \leq A^{*}-M^{*}\right) U^{*} d v\right] \\
& =E\left[\int_{-M^{*}}^{A^{*}-M^{*}} 1 d v U^{*}\right]=E\left[\left(A^{*}-M^{*}+M^{*}\right) U^{*}\right] \\
& =E\left(A^{*} U^{*}\right)=E\left(A^{*}\right) E\left(U^{*}\right)
\end{aligned}
$$

and by the same logic

$$
E(W)=E\left(\frac{D}{f(V \mid X)}\right)=E\left(A^{*}\right)
$$


so

$$
p \lim \frac{\sum_{i=1}^{n} U_{i} W_{i}}{\sum_{i=1}^{n} W_{i}}=\frac{E(U W)}{E(W)}=\frac{E\left(A^{*}\right) E\left(U^{*}\right)}{E\left(A^{*}\right)}=E\left(U^{*}\right)
$$

Now consider the case where $A^{*}$ is infinity. In that case $E(U W)$ and $E(W)$ are both infinite. To deal with this complication, define

$$
\begin{gathered}
W_{\tau i}=\frac{I\left(V_{i} \leq \tau\right) W_{i}}{\tau}=\frac{I\left(V_{i} \leq \tau\right) D_{i}}{\tau f\left(V_{i} \mid X_{i}\right)} \\
\widehat{\mu}_{\tau 1}=\frac{1}{n} \sum_{i=1}^{n} U_{i} W_{\tau i}, \quad \widehat{\mu}_{\tau 2}=\frac{1}{n} \sum_{i=1}^{n} W_{\tau i}
\end{gathered}
$$

where $\tau=\tau(n)$ is an asymptotic trimming parameter. Let $\tau \rightarrow \infty$ at a rate that makes $\left[\inf _{x \operatorname{supp}(X)} F(\tau \mid x)\right]^{n} \rightarrow 1$ where $F$ is the cumulative distribution function of $V \mid X$. By the definition of $\widehat{\mu}_{\tau 1}$,

$$
\begin{aligned}
\operatorname{Pr}\left(\widehat{\mu}_{\tau 1}=\frac{1}{n \tau} \sum_{i=1}^{n} U_{i} W_{i}\right) & =\operatorname{Pr}\left(\frac{1}{n \tau} \sum_{i=1}^{n} U_{i} W_{i} I\left(V_{i}>\tau_{i}\right)=0\right) \\
& \leq \prod_{i=1}^{n} \operatorname{Pr}\left(V_{i} \leq \tau\right) \\
& \leq \prod_{i=1}^{n} X, U^{*}, M^{*}, A^{*} \in \operatorname{supp}\left(X, U^{*}, M^{*}, A^{*}\right) \\
& \leq\left[\inf _{x \operatorname{supp}(X)} F(\tau \mid x)\right]^{n} \rightarrow 1
\end{aligned}
$$

so $\widehat{\mu}_{\tau 1}-(n \tau)^{-1} \sum_{i=1}^{n} U_{i} W_{i} \rightarrow 0$ with probability one. The same logic replacing $U_{i}$ with one shows that $\widehat{\mu}_{\tau 2}-(n \tau)^{-1} \sum_{i=1}^{n} W_{i} \rightarrow 0$ with probability one, and therefore $\left(\widehat{\mu}_{\tau 1} / \widehat{\mu}_{\tau 2}\right)-\widehat{\mu} \rightarrow 0$ with probability one, given the fast rate that $\tau \rightarrow$ $\infty$. It follows that

$$
p \lim \widehat{\mu}=p \lim \frac{\widehat{\mu}_{\tau 1}}{\widehat{\mu}_{\tau 2}}=\lim _{\tau \rightarrow \infty} \frac{E\left(\widehat{\mu}_{\tau 1}\right)}{E\left(\widehat{\mu}_{\tau 2}\right)}
$$


assuming $E\left(\widehat{\mu}_{\tau 1}\right)$ and $E\left(\widehat{\mu}_{\tau 2}\right)$ are finite for any given sufficiently large $\tau$. Now

$$
\begin{aligned}
& E\left(\widehat{\mu}_{\tau 1}\right)=E\left(\frac{I(V \leq \tau) D U^{*}}{\tau f(V \mid X)}\right) \\
& =E\left[E\left(\frac{I\left(0 \leq M^{*}+V\right) I(V \leq \tau) U^{*}}{\tau f(V \mid X)} \mid X, U^{*}, M^{*}\right)\right] \\
& =E\left[\int_{\operatorname{supp}\left(V \mid X, U^{*}, M^{*}\right)} \frac{I\left(-M^{*} \leq V \leq \tau\right) U^{*}}{\tau f(V \mid X)} f\left(V \mid X, U^{*}, M^{*}\right) d v\right] \\
& =E\left[\int_{\operatorname{supp}(V \mid X)} \frac{I\left(-M^{*} \leq V \leq \tau\right) U^{*}}{\tau} d \nu\right] \\
& =E\left[\int_{-M^{*}}^{\tau} \frac{1}{\tau} d v U^{*}\right] \\
& =E\left(U^{*}\right)+\frac{E\left(M^{*} U^{*}\right)}{\tau}
\end{aligned}
$$

and by the same logic

$$
E\left(\widehat{\mu}_{\tau 2}\right)=1+\frac{E\left(M^{*}\right)}{\tau}
$$

so $\lim _{\tau \rightarrow \infty} E\left(\widehat{\mu}_{\tau 1}\right) / E\left(\widehat{\mu}_{\tau 2}\right)=E\left(U^{*}\right)$.

The remaining case to consider is where $A^{*}$ is random and has infinite mean. This can include the case where $A^{*}$ has a positive but less than one probability of being infinite, corresponding to the case where some fraction of the population has selection that is not bounded from above. For this case, consider the more complicated weighting function $W_{\tau i}^{*}$ defined by

$$
W_{\tau i}^{*}=\frac{I\left(V_{i} \leq \tau\right) W_{i}}{\left(1-I_{i}^{*}\right) \tau+I_{i}^{*} A_{i}^{*}}
$$

where $I_{i}^{*}$ equals one if $A_{i}^{*}$ is finite and zero otherwise, and assume $\tau \rightarrow \infty$ sufficiently fast so that $\operatorname{prob}\left(\tau<A_{i}^{*}-M_{i}^{*}\right)$ is zero when $I_{i}^{*}=1$. Following the same logic as before

$E\left(\frac{I(V \leq \tau) W}{\left(1-I^{*}\right) \tau+I^{*} A^{*}}\right)=E\left(\frac{I(V \leq \tau) D U^{*}}{\left[\left(1-I^{*}\right) \tau+I^{*} A^{*}\right] f(V \mid X)}\right)$ 


$$
\begin{aligned}
& =E\left[\int_{\operatorname{supp}\left(V \mid X, U^{*}, M^{*}, A^{*}\right)} \frac{I\left(-M^{*} \leq V \leq \min \left(\tau, A^{*}-M^{*}\right)\right) U^{*}}{\left[\left(1-I^{*}\right) \tau+I^{*} A^{*}\right] f(V \mid X)} f\left(V \mid X, U^{*}, M^{*}, A^{*}\right) d v\right] \\
& =E\left[\int_{-M^{*}}^{\min \left(\tau, A^{*}-M^{*}\right)} \frac{1}{\left(1-I^{*}\right) \tau+I^{*} A^{*}} d v U^{*}\right] \\
& =E\left[I^{*} \int_{-M^{*}}^{A^{*}-M^{*}} \frac{1}{A^{*}} d v U^{*}+\left(1-I^{*}\right) \int_{-M^{*}}^{\tau} \frac{1}{\tau} d v U^{*}\right] \\
& =E\left[I^{*} U^{*}+\left(1-I^{*}\right) U^{*}+\frac{\left(1-I^{*}\right) M^{*} U^{*}}{\tau}\right] \\
& =E\left(U^{*}\right)+\frac{E\left[\left(1-I^{*}\right) M^{*} U^{*}\right]}{\tau}
\end{aligned}
$$

and the remainder of the proof also follows as before.

The proof of Corollary 1 is omitted to save space, since it follows the same logic as the proof of Theorem 1 in the case where $A^{*}$ is infinite, with a fixed instead of asymptotic $\tau$.

Proof of Corollary 2. Let $\bar{W}$ and $\overline{W U}$ denote the sample means of $W_{i}$ and $W_{i} U_{i}$, respectively, and let $c=\sup (\operatorname{supp}(W))$. Then $E\left((W U)^{2}\right)=$ $E\left(\left(W U^{*}\right)^{2}\right) \leq c^{2} E\left(U^{*}\right)^{2}$, and similarly $E\left((W)^{2}\right) \leq c^{2}$ so $W_{i}$ and $W_{i} U_{i}$ have finite second moments. Assumption A1 also implies $E(W)>0$. Corollary 2 then follows from applying the Lindeberg-Levy central limit theorem to $(\overline{W U}, \bar{W})$, and the delta method.

Proof of THEOREM 2. $\theta_{0}$ is equivalently given by

$$
\theta_{0}=\arg \min _{\theta \in \Theta}\left(\frac{E[W \psi(P, X, V, \theta)]}{E(W)}\right)^{\prime} \Omega\left(\frac{E[W \psi(P, X, V, \theta)]}{E(W)}\right)
$$

The first order condition for $\theta_{0}$ and the mean value theorem give

$$
\begin{aligned}
0 & =v\left(\theta_{0}\right)^{\prime} \Omega \frac{E\left[W \psi\left(P, X, V, \theta_{0}\right)\right]}{E(W)} \\
& =v\left(\theta_{0}\right)^{\prime} \Omega\left(\frac{E\left[W \psi\left(P, X, V, \theta^{*}\right)\right]}{E(W)}+v(\widetilde{\theta})\left(\theta_{0}-\theta^{*}\right)\right) \\
& =v\left(\theta_{0}\right)^{\prime} \Omega\left[\mu+v(\widetilde{\theta})\left(\theta_{0}-\theta^{*}\right)\right]
\end{aligned}
$$


where $\widetilde{\theta}$ lies between $\theta^{*}$ and $\theta_{0}$. Solving for $\theta_{0}-\theta^{*}$ gives

$$
\theta_{0}-\theta^{*}=-\left[v\left(\theta_{0}\right)^{\prime} \Omega v(\widetilde{\theta})\right]^{-1} v\left(\theta_{0}\right)^{\prime} \Omega \mu
$$

Now $E\left(U^{*}\right)=0$, so if $\mu=E\left(U^{*}\right)$ then $\theta_{0}-\theta^{*}=0$, while if $\mu=E\left(U^{*}\right)+$ $O\left(\tau^{-1}\right)$ then

$$
\theta_{0}-\theta^{*}=-\left[v\left(\theta_{0}\right)^{\prime} \Omega v(\widetilde{\theta})\right]^{-1} v\left(\theta_{0}\right)^{\prime} \Omega O\left(\tau^{-1}\right)=O\left(\tau^{-1}\right)
$$

Proof of COROLlary 3. This is standard GMM limiting distribution theory with iid data. See, e.g., Newey and McFadden (1984) or Wooldridge (2001) Theorems 14.1 and 14.2.

\section{References}

[1] ABADIE, A., (2001), "Semiparametric Instrumental Variable Estimation of Treatment Response Models," Unpublished Manuscript, Harvard University.

[2] Abel, A. B. And J. C. Eberly, (1994) "A Unified Model of Investment Under Uncertainty," American Economic Review, 84, 1369-1384.

[3] Ahn, AND J. L. Powell, (1993), "Semiparametric Estimation of Censored Models with a Nonparametric Selection Mechanism," Journal of Enometrics, 58, 3-29.

[4] Anton, A. A., S. A. Fernández, And J. Rodriguez-Póo (2001), "Semiparametric Estimation of a Duration Model," Oxford Bulletin of Economics \& Statistics, 63, 517-533.

[5] Andrews, D. W. K. And M. M. A. Schafgans (1998), "Semiparametric Estimation of the Intercept of a Sample Selection Model," Review of Economic Studies, 65, 497-517.

[6] Angrist, J. And G. Imbens, (1995), "Two-Stage Least Squares Estimation of Average Causal Effects in Models With Variable Treatment Intensity," Journal of the American Statistical Association 90, 430-442. 
[7] Blundell, R., S. Bond, And C. Meghir (1996), "Econometric Models of Company Investment," in The econometrics of panel data: A handbook of the theory with applications. Matyas, Laszlo Sevestre, Patrick, eds., Second edition, London: Kluwer Academic. 685-710.

[8] Blundell, R. And J. L. Powell (2004), "Endogeneity in Semiparametric Binary Response Models," Review of Economic Studies, 71, 655-679.

[9] Cameron, S. V., And J. J. Heckman. (1998), "Life Cycle Schooling and Dynamic Selection Bias: Models and Evidence for Five Cohorts of American Males," Journal of Political economy 106, 262-333.

[10] Carneiro, P., K. T. Hansen, And J. J. Heckman (2003), "Estimating Distributions of Treatment Effects With an Application to the Returns to Schooling," International Economic Review, 44, 361-422.

[11] Carneiro, P., And J. J. Heckman (2002), "The Evidence on Credit Constraints in Post-secondary Schooling," Economic Journal, 112, 705-734.

[12] Cavanagh, C. And R. P. Sherman (1998), "Rank Estimators for Monotonic Index Models", Journal of Econometrics, 84, 351-381

[13] Chen, S. H., (2003), "Estimating Wage Volatilities for College versus High School Careers," SUNY Albany unpublished manuscript.

[14] Chen, S. H., AND S. KHAN, (2003), "Nonparametric Estimation of Volatility Differentials in Selection Models with an Application to Returns to Schooling," SUNY Albany unpublished manuscript.

[15] Chen, S. And L. F. LeE. (1998), "Efficient Semiparametric Scoring of Sample Selection Models," Econometric Theory, 14, 423-462.

[16] CHOI, K. (1990) The Semiparametric Estimation of the Sample Selection Model Using Series Expansion and the Propensity Score," University of Chicago manuscript.

[17] Cogneau, D. And E. Maurin, (2001), Parental Income and School Attendence in a Low-Income Country: a Semi-parametric Analysis," Unpublished Manuscript. 
[18] Cosslett, S. R. (1991), "Semiparametric Estimation of a Regression Model with Sample Selectivity," in W. A. Barnett, J. L. Powell, and G. Tauchen, eds., Nonparametric and Semiparametric Methods in Econometrics and Statistics. Cambridge: Cambridge University Press.

[19] Das, M., W. K. Newey, AND F. Vella (2003), "Nonparametric estimation of sample selection models," Review of Economic Studies, 70, 33-58.

[20] Doms, M. And T. Dunne, (1998), "Capital Adjustment Patterns in Manufacturing Plants," Review of Economic Dynamics, 1, 409-429.

[21] Donald, S. (1995), "Two Step Estimation of Heteroskedastic Sample Selection Models," Journal of Econometrics, 65, 347-380.

[22] Eisner, R. And R. Strotz, (1963) 'Determinent of Investment Behavior," in Impact of Monetary Policy. Englewood Cliffs,NJ: Prentice-Hall.

[23] Gronau, R. (1974), "Wage Comparisons - A Selectivity Bias," Journal of Political Economy, 82, 1119-1144.

[24] HAN, A. K. (1987) "Non-parametric Analysis of a Generalized Regression Model," Journal of Econometrics, 35, 303-31.

[25] Hahn, J. (1998), On the Role of The Propensity Score in Efficient Semiparametric Estimation of Average Treatment Effects, Econometrica, 66, 315-331.

[26] Hayashi, F., (1982) 'Tobin's Marginal q and Average q: A Neoclassical Interpretation." Econometrica, 50, 213-224.

[27] Heckman, J. (1974), "Shadow Prices, Market Wages, and Labor Supply," Econometrica, 42, 679-693

[28] Heckman, J. (1976), "The Common Structure of Statistical Models of Truncation, Sample Selection and Limited Dependent Variables and a Simple Estimator for Such Models," Annals of Economic and Social Measurement, 5, 475-495

[29] Heckman, J. (1976), "Sample Selection Bias as a Specification Error," Econometrica, 47, 153-161. 
[30] Heckman, J. (1990), "Varieties of Selection Bias," American Economic Review: Papers and Proceedings, 313-318.

[31] Heckman, J., H. Ichimura, And P. Todd (1998), Matching as an Econometric Evaluation Estimator, Review of Economic Studies, 65, 261294.

[32] Heckman, J. And E. Vytlacil (2004), "Structural Equations, Treatment Effects, and Econometric Policy Evaluation," unpublished manuscript.

[33] Hirano, K., G. W. Imbens and G. Ridder, (2003), "Efficient Estimation of Average Treatment Effects Using the Estimated Propensity Score," Econometrica 71, 1161-1189.

[34] Hogan, V. And R. Rigobon (2003), "Using Unobserved Supply Shocks to Estimate the Returns to Educations," Unpublished manuscript.

[35] Horowitz, J. L., (1992), "A Smoothed Maximum Score Estimator for the Binary Response Model”, Econometrica, 60, 505-531.

[36] Horvitz, D.G. And D. J. Thompson, (1952), "A generalization of sampling without replacement from a finite universe" Journal of the American Statistical Association, 47, 663-685.

[37] IChimura, H. And L. F. LeE (1991), "Semiparametric Least Squares Estimation of Multiple Index Models: Single Equation Estimation," in W. A. Barnett, J. L. Powell, and G. Tauchen, eds., Nonparametric and Semiparametric Methods in Econometrics and Statistics. Cambridge: Cambridge University Press.

[38] Imbens, G. W., AND J. ANGRIST (1994), Identification and Estimation of Local Average Treatment Effects, Econometrica, 62, 476-476.

[39] Jacho-Chavez, D. T. (2005), "Efficiency Bounds in Semiparametric Models defined by Moment Restrictions using an Estimated Conditional Probability Density, " unpublished manuscript.

[40] Kane, T. J. And C. E. Rouse (1995), "Labor-Market Returns to Two and Four-Year College," American Economic Review 85, 600-614.

[41] Koul, H. L., V. Susarla AND J. VAn RyZIN (1981), "Regression Analysis With Randomly Right Censored Data," Annals of Statistics 9, 1276-1288. 
[42] Khan, S. And A. Lewbel (2005), "Weighted and Two Stage Least Squares Estimation of Semiparametric Truncated Regression Models," unpublished manuscript.

[43] Kyriazidou, E. (1997), "Estimation of a Panel Data Sample Selection Model," Econometrica 65, 1334-1364

[44] LEE, L. F. (1982), "Some Approaches to the Correction of Selectivity Bias," Review of Economic Studies, 49, 355-372.

[45] LEE, L. F. (1992), "Semiparametric Two Stage Estimation of Sample Selection Models Subject to Tobit-type Selection Rules," Journal of Econometrics, 61, 305-344.

[46] LEE, L. F. (1994), "Semiparametric Instrumental Variables Estimation of Simultaneous Equation Sample Selection Models," Journal of Econometrics, 63, 341-388.

[47] LewBel, A. (1998), "Semiparametric Latent Variable Model Estimation With Endogenous or Mismeasured Regressors," Econometrica, 66, 105121.

[48] LewBEL, A. (2000), "Semiparametric Qualitative Response Model Estimation With Unknown Heteroscedasticity or Instrumental Variables," Journal of Econometrics, 97, 145-177.

[49] Lewbel, A., O. Linton, And D. McFadden (2001), "Estimating Features of a Distribution From Binomial Data," Unpublished manuscript.

[50] Lewbel, A., And S. Schennach (2005), "A Simple Ordered Data Estimator for Inverse Density Weighted Expectations," Journal of Econometrics, forthcoming

[51] Magnac, T. And E. Maurin. (2003), "Identification and Information in Monotone Binary Models," unpublished manuscript.

[52] Manski, C. F. (1975), "Maximum Score Estimation of the Stochastic Utility Model of Choice", Journal of Econometrics, 3, 205-228

[53] Manski, C. F. (1985), "Semiparametric Analysis of Discrete Response: Asymptotic Properties of Maximum Score Estimation”, Journal of Econometrics, 27, 313-334. 
[54] Manski, C. F. (1994), "The Selection Problem," In Sims, C. Ed., Advances in Econometrics, Cambridge: Cambridge University Press.

[55] Maurin, E. (1999), "The Impact of Parental Income on Early Schooling Transitions: A Re-examination Using Data Over Three Generations," CREST-INSEE unpublished manuscript.

[56] McFadDEn, D. L. (1984), "Econometric Analysis of Qualitative Response Models," Handbook of Econometrics, vol. 2, ed. by Z. Griliches and M. D. Intriligator, pp. 1395-1457, Amsterdam: Elsevior.

[57] Newey, W. K. (1984), "A Method of Moments Interpretation of Sequential Estimators," Economics Letters, 14, 201-206.

[58] Newey, W. K. (1988), ’Two Step Estimation of Sample Selection Models," Princeton University manuscript.

[59] Newey, W. K. (1999), "Consistency of Two-Step Sample Selection Estimators Despite Misspecification of Distribution," Economics Letters, 63, 129-132.

[60] Newey, W. K. And D. McFadden (1994), "Large Sample Estimation and Hypothesis Testing," in Handbook of Econometrics, vol. iv, ed. by R. F. Engle and D. L. McFadden, pp. 2111-2245, Amsterdam: Elsevier.

[61] Nilsen, O. A. And F. Schiantarelli (2003), "Zeros And Lumps In Investment: Empirical Evidence On Irreversibilities And Nonconvexities," The Review of Economics and Statistics, 85, 1021-1037.

[62] Powell, J. L., (1994), "Estimation of Semiparametric Models," Handbook of Econometrics, vol. 4, ed. by R. F. Engle and D. L. McFadden, pp. 24432521, Amsterdam: Elsevior.

[63] Powell, J. L., J. H. Stock, And T. M. Stoker (1989), "Semiparametric Estimation of Index Coefficients," Econometrica 57, 1403-1430.

[64] Rubin, D. (1974), Estimating Causal Effects of Treatments in Randomized and Nonrandomized Studies, Journal of Educational Psychology, 66, 688701. 
[65] Rosenbaum, P. And D. Rubin, (1985), "Reducing Bias in Observational Studies Using Subclassification on the Propensity Score," Journal of the American Statistical Association, 79, 516-524.

[66] Summers, L. H. (1981), "Taxation and Corporate Investment: A q-Theory Approach," Brookings Papers on Economic Activity, 1, 67-127.

[67] TChamourliYski, Y. (2002), "Returns to Experience and Seniority" Evidence From a Panel Selection Model With Endogenous Regressors and No Exclusion Restriction,” Boston College Unpublished Manuscript.

[68] Vella, F. (1998), "Estimating Models With Sample Selection Bias: A Survey," Journal of Human Resources, 33, 127-169.

[69] Vytlacil, E. (2002), "Independence, Monotonicity, and Latent Index Models: An Equivalence Result," Econometrica, 70, 331-341.

[70] Wainer, H. (1986), Drawing inferences from self-selected samples, New York: Springer-Verlag.

[71] WoOldRidge, J. M. (1995), "Selection Corrections for Panel Data Models Under Conditional Mean Independence Assumptions," Journal of Econometrics, 68, 115-132.

[72] Wooldridge, J. M. (2001), Econometric Analysis of Cross Section and Panel Data, Cambridge: MIT Press. 
Table 1. Estimates of the Outcome Equation Profit Coefficient

\begin{tabular}{lllllll} 
& \multicolumn{2}{c}{ no dummies } & \multicolumn{2}{c}{ plant type dummies } & \multicolumn{2}{c}{ types \& ISIC dummies } \\
OLS & .231 & .036 & .219 & .035 & .221 & .035 \\
2SLS & .383 & .051 & .353 & .050 & .355 & .050 \\
Heckman & .298 & .087 & .287 & .092 & .298 & .094 \\
Endogenous ML & .468 & .061 & .403 & .062 & .413 & .057 \\
Weighted OLS & .323 & .062 & .317 & .059 & .316 & .051 \\
Weighted TSLS & .470 & .070 & .431 & .073 & .411 & .080
\end{tabular}

Notes: In each block, the first number is $\beta_{1}$, the coefficient of the profit rate in the outcome equation, and the second number is the estimated standard error. In the first pair of columns, $X_{2}$ and $Z_{2}$ consist only of the constant term. In the second pair of columns, $X_{2}$ and $Z_{2}$ also include plant type dummies, and in the third pair of columns, $X_{2}$ and $Z_{2}$ contain dummies both for plant type and for two digit industry (ISIC) code.

Table 2. Simulations of the Outcome Equation Profit Coefficient

$\begin{array}{lllllllllll} & \text { MEAN } & \text { SD } & \text { LQ } & \text { MED } & \text { UQ } & \text { RMSE } & \text { MAE } & \text { MDAE } & \text { MESE } & \% 2 S E \\ \text { OLS } & .231 & .017 & .221 & .232 & .245 & .183 & .182 & .182 & .036 & .000 \\ \text { TSLS } & .362 & .023 & .346 & .362 & .377 & .056 & .052 & .051 & .051 & .987 \\ \text { Heckman ML } & .268 & .020 & .254 & .268 & .282 & .146 & .145 & .145 & .102 & .997 \\ \text { Endogenous ML } & .414 & .028 & .394 & .413 & .433 & .028 & .023 & .020 & .145 & 1.00 \\ \text { Weighted OLS } & .243 & .043 & .213 & .243 & .273 & .177 & .171 & .170 & .049 & .060 \\ \text { Weighted TSLS } & .399 & .061 & .358 & .397 & .440 & .063 & .050 & .043 & .066 & .961\end{array}$

Notes: In these simulations the 'true' value of the coefficient is .4132. The reported statistics are as follows. MEAN and SD are the mean and standard deviation of the estimates across the simulations. LQ, MED, and UQ are the $25 \%$ (lower) $50 \%$ (median) and $75 \%$ (upper) quartiles. RMSE, MAE, and MDAE are the root mean squared error, mean absolute error and median absolute error of the estimates. MESE is the mean estimated standard error, and \%2SE is the percentage of simulations in which the true coefficient was within two estimated standard errors of the estimated coefficient. 
Table 3. Estimates of Wage Outcome Equations

\begin{tabular}{|c|c|c|c|c|c|c|}
\hline \multirow[b]{2}{*}{ Constant } & \multicolumn{2}{|c|}{ OLS } & \multicolumn{2}{|c|}{ MLE } & \multicolumn{2}{|c|}{ Kernel Weighted OLS } \\
\hline & 1.37 & .053 & 1.69 & .111 & 1.40 & .371 \\
\hline Test Score & .120 & .009 & .059 & .020 & .109 & .061 \\
\hline Parent College & .048 & .016 & -.048 & .034 & -.011 & .122 \\
\hline Black & -.017 & .027 & -.087 & .035 & -.047 & .140 \\
\hline Male & .249 & 015 & .266 & .017 & .225 & .113 \\
\hline Urban & .161 & 020 & 132 & .023 & 167 & .104 \\
\hline 90's cohort & .097 & .018 & .086 & .019 & .122 & .117 \\
\hline 1 to 5 years work & .001 & .038 & -.000 & .038 & .027 & .273 \\
\hline Over 5 years & .342 & .051 & .337 & .051 & .350 & .306 \\
\hline & Norma & Weighted GMM & Sorted & Weighti & & \\
\hline Constant & 1.34 & .143 & 1.51 & .132 & & \\
\hline Test Score & .161 & .027 & .129 & .022 & & \\
\hline Parent College & .118 & .058 & .027 & .064 & & \\
\hline Black & -.162 & .079 & -.135 & .103 & & \\
\hline Male & -.077 & .053 & .167 & .056 & & \\
\hline Urban & .019 & .063 & .054 & .062 & & \\
\hline 90's cohort & .207 & .076 & .152 & .073 & & \\
\hline 1 to 5 years work & .198 & .057 & -.047 & .084 & & \\
\hline Over 5 years & .194 & .087 & .222 & .091 & & \\
\hline
\end{tabular}

Notes: In each block, the first number is the coefficient, and the second number is the estimated standard error. OLS is the wage equation only using data on college graduates with no graduate education, and so does not control for any selection bias. ML is a parametric two equation system of selection and wages with normal errors. Weighted OLS is the density weighted semiparametric estimator of the wage equation, using a kernel estimator of the conditional density of $V$ given $X$. Normal Weighted GMM models $V$ as linear in $X$ with an independent normal error, estimating the $V$ and density weighted wage equations simultaneously by GMM. Sorted Weighting OLS models $V$ as linear in $X$ with an independent error of unknown density that is estimated using the numerically trivial sorted data estimator. 